\title{
Extended Assessment of Stability and Control Prediction Methods for NATO Air Vehicles: Summary
}

\author{
Adam Jirásek* and Russell M. Cummings $₫$ \\ U.S. Air Force Academy, Colorado 80840 \\ and \\ Andreas Schütte \\ DLR, German Aerospace Center, 38108 Brunswick, Germany
}

DOI: $10.2514 / 1 . C 033818$

\begin{abstract}
This paper presents cross comparisons and assessments of the aerodynamic results obtained within the North Atlantic Treaty Organization's Science and Technology Organisation Applied Vehicle Technology-201 Task Group. The results of five participating organizations and six different computational fluid dynamics solvers are compared to the available wind-tunnel data from the German-Dutch Wind Tunnels, and the results were obtained at low subsonic speeds for both static and dynamic cases. The comparisons help to find commonalities and disparities in the computational fluid dynamics predictions of the aerodynamics of a generic unmanned combat aerial vehicle (the stability and control configuration) and point to areas where computational fluid dynamics is challenged by nonlinear aerodynamics flow prediction. These comparisons help to show where the modeling and simulation of novel aircraft platforms in the earliest stages of design development can contribute to a more successful design process by determining difficult nonlinear aerodynamics and helping to change the design before the later stages of the design process.
\end{abstract}

$\begin{array}{ll} & \text { Nomenclature } \\ a & =\text { speed of sound } \\ C_{D} & =\text { drag coefficient } \\ C_{L} & =\text { lift coefficient } \\ C_{M_{x}} & =\text { roll moment coefficient } \\ C_{M_{y}} & =\text { pitch moment coefficient } \\ C_{M_{z}} & =\text { yaw moment coefficient } \\ C_{N} & =\text { normal force coefficient } \\ C_{Y} & =\text { side force coefficient } \\ d c & =\text { drag count, equal to } 0.0001 \\ f & =\text { frequency, Hz } \\ M & =\text { Mach number; } v / a \\ R e & =\text { Reynolds number } \\ v & =\text { velocity } \\ \alpha & =\text { angle of incidence } \\ \beta & =\text { angle of sideslip } \\ \phi & =\text { basis function } \\ \Psi & =\text { yaw angle }\end{array}$

\section{Introduction}

$\mathbf{H}$ ISTORICALLY, stability and control (S\&C) engineers have used an iterative process combining semiempirical, lowerorder, wind-tunnel, and flight-test modeling techniques to determine the aerodynamic characteristics of new fighter aircraft. Despite their greatest efforts using the best available predictive capabilities, nearly every major fighter program since 1960 has had costly nonlinear aerodynamic or fluid-structure interaction issues that were not discovered until flight testing [1는. Some examples include the

Presented as Paper 2014-2394 at the 32nd AIAA Applied Aerodynamics Conference, Atlanta, GA, 16-20 June 2014; received 10 August 2016; revision received 18 November 2017; accepted for publication 15 December 2017; published online 29 January 2018. This material is declared a work of the U.S. Government and is not subject to copyright protection in the United States. All requests for copying and permission to reprint should be submitted to CCC at www.copyright.com; employ the ISSN 0021-8669 (print) or 1533-3868 (online) to initiate your request. See also AIAA Rights and Permissions www.aiaa.org/randp.

*Affiliated Research Associate, Department of Aeronautics. Senior Member AIAA.

${ }^{\dagger}$ Professor of Aeronautics, Department of Aeronautics. Fellow AIAA.

*Research Scientist, Institute of Aerodynamics and Flow Technology. Senior Member AIAA.

${ }^{\S}$ Research Scientist, Institute of Aerodynamics and Flow Technology.
F-15 [5] , F/A-18A [5] , F/A-18C [6], AV-8B [5] ], and the B-2 bomber [7]. The F-15, F/A $-18 \mathrm{~A}$, and $\mathrm{AV}-8 \mathrm{~B}$ all exhibited significant aeroelastic flutter [5]; whereas the F/A-18C experienced tail buffet at high angles of attack due to leading-edge extension vortex breakdown [6], and the B-2 bomber experienced a residual pitch oscillation [7] ]. The development costs of each of these aircraft could have been drastically reduced if these issues had been identified earlier in the design process. However, existing semiempirical lowerorder modeling and wind-tunnel techniques are incapable of reliably predicting unsteady nonlinear aerodynamic behavior over the full flight envelope. Clearly, a high-fidelity computational tool capable of reliably predicting and/or identifying configurations susceptible to handling quality instabilities before flight testing would be of great interest to the S\&C community. Such a tool is well suited to the aircraft design phase and would decrease the cost and risks incurred by flight-testing and post-design-phase modifications. The vision of using computational fluid dynamics (CFD) in the initial aircraft design initiated several projects within S\&C CFD and the windtunnel community, such as the Computational Methods for Stability and Control [3] and North Atlantic Treaty Organization's (NATO's) Science and Technology Organisation (STO) Applied Vehicle Technology (AVT)-161 Task Group, titled "Assessment of Stability and Control Prediction Methods for NATO Air and Sea Vehicles" [8].

The modern design of new combat aircraft for which the driving factor is the aircraft combat survivability [9] requires new platforms where low observability and higher maneuverability makes the problem of nonlinear aerodynamic characteristics and their predictions more urgent then ever. The low observability requirement leads to unconventional designs with swept leading and trailing edges, and high maneuverability requirement leads to flight regimes at high angles of incidence and high turn.

This paper presents common cross comparisons of the CFD results obtained by several participants within the NATO Science and TechnologyOrganisation's AVT-201 Task Group (titled Extended Assessment of Reliable Stability and Control Prediction Method for NATO Air and Sea Vehicles) [10-30]. The objective of the group is to create an overall strategy for creating $\mathrm{S} \& \mathrm{C}$ databases for vehicle simulations at full-scale conditions, including deflection of control surfaces, throughout the operational envelope of the vehicle.

The vehicle used for the study is an aircraft denoted DLR-F17 that has a shape typical for modern high-performance unmanned aerial vehicles [10-12], as shown in Fig. 1. This geometry was used previously in the NATO RTO Task Group AVT-161 [31] and contains many details typical for this type of aircraft: combination 


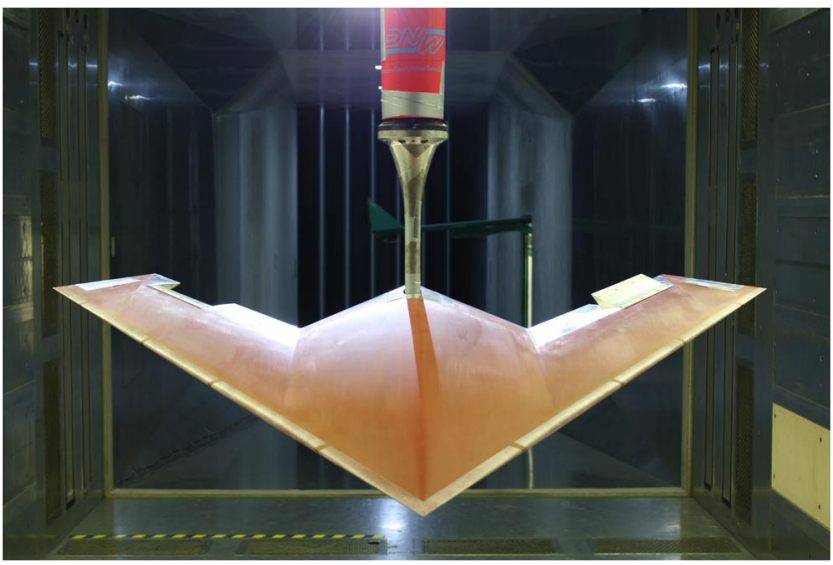

Fig. 1 SACCON geometry with control surfaces in DNW-NWB wind tunnel; image courtesy of DLR.

of sharp and blunt leading edges; swept leading and trailing edges; and strongly nonlinear characteristics, as was found for the original stability and control configuration (SACCON) configuration, as shown in Fig. 1 in the German-Dutch Wind Tunnels (DNW-NWB). The flow physics characteristics of this aircraft are already well known and have been discussed in detail [32]. The configuration has now been tested in low-speed wind tunnels at several wind-tunnel laboratories $[\underline{12}, 13]$, as well as in high-speed wind tunnels [14] using a different model.

The intention of Task Group AVT-201 is to be able to predict aerodynamic nonlinearities (such as those found in the SACCON pitching moment) during the early stages of the design process before the aircraft details are fixed to such an extent that any change due to unexpected behavior represents a major cost factor during the aircraft development process. To achieve this goal, the participants selected a number of common cases, which are analyzed in great detail, and the predictions are compared to wind-tunnel data. The available predictions are compared with each other in this paper in order to determine where CFD codes and turbulence models are being challenged by the nonlinear aerodynamics of this class of aircraft.

\section{List of Participants and Description of CFD Solvers}

The list of participants in this CFD assessment study is shown in Table 1 [33], which also lists the participating organizations, the names

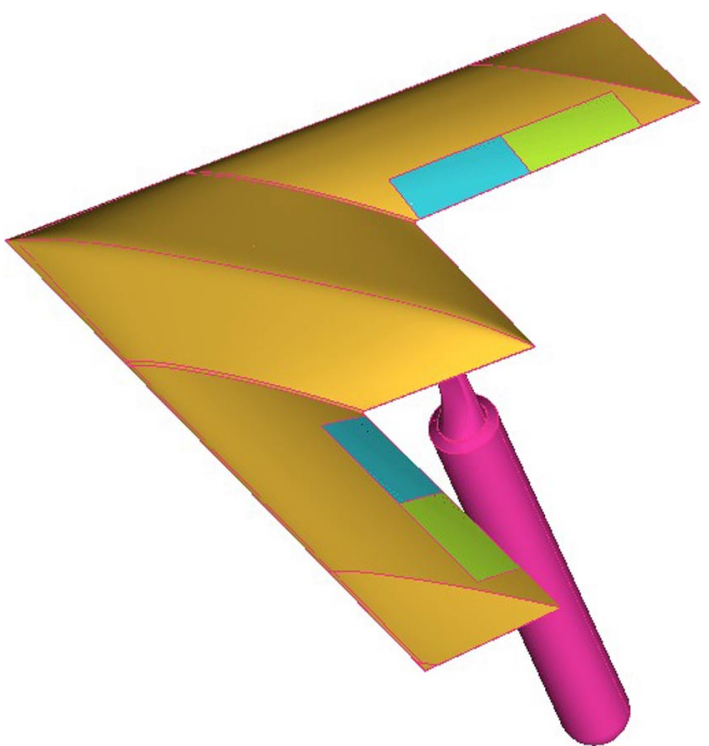

Fig. 2 SACCON geometry including trailing-edge control surfaces and the sting.

of the solvers, and the general solver characteristics. For more details about each code, the reader is referred to the references listed next to the names of the respective organizations in the table. Some of the major differences in the approaches of the participants that may contribute to differences between CFD results are also mentioned in the table.

\section{DLR, German Aerospace Center's F-17 Computational Model Geometries}

The wind-tunnel results used in the common comparisons in this paper were obtained in the German-Dutch Wind Tunnels' LowSpeed Wind Tunnel $[12,13,29]$. The German Dutch Wind TunnelsNiedergeschwindigkeits-Windkanal Braunschweig (DNW-NWB) belongs to the foundation of German-Dutch Wind Tunnels under Dutch law. The DNW operates 12 different wind tunnels on five sites in Germany and The Netherlands. The DNW-NWB is located on the DLR, German Aerspace Center (DLR) site in Braunschweig, Germany. It is a closed-circuit atmospheric-type wind tunnel, which can be operated with either an open, slotted, or closed test section. The test section size is 3.25 by $2.8 \mathrm{~m}$ ( 10.6 by $9.2 \mathrm{ft})$. The maximum

Table 1 Overview of organizations and CFD codes

\begin{tabular}{|c|c|c|c|c|c|c|}
\hline $\begin{array}{l}\text { Name of contributing } \\
\text { organization }\end{array}$ & $\begin{array}{l}\text { CFD } \\
\text { solver }\end{array}$ & $\begin{array}{l}\text { Structured vs } \\
\text { unstructured }\end{array}$ & Turbulence model & $\begin{array}{l}\text { Sting } \\
\text { geometry }\end{array}$ & Additional notes & Legend in figures \\
\hline DLR [16] & TAU & Unstructured & DRSM & Yes & -- & DLR-RSM \\
\hline FOI $[\underline{2 \overline{8}}]$ & Edge & Unstructured & EARSM & Yes & -- & FOI-EARSM \\
\hline FOI $[\overline{28}]$ & Edge & Unstructured & $\mathrm{SA}$ & Yes & -- & FOI-SA \\
\hline FOI $[\overline{28}]$ & Edge & Unstructured & SST & Yes & -- & FOI-SST \\
\hline $\begin{array}{l}\text { NASA Langley Research } \\
\text { Center [17] }\end{array}$ & USM3D & Unstructured & SA & Yes & -- & NASA-SA \\
\hline $\begin{array}{l}\text { NASA Langley Research } \\
\text { Center [17] }\end{array}$ & USM3D & Unstructured & SST & Yes & -- & NASA-SST \\
\hline University of Liverpool [18] & PMB & Structured & $\begin{array}{l}k-\omega \text { with Brandsma's } k \\
\text { vortex core limiter [33] }\end{array}$ & No & $\begin{array}{l}\text { Blending surface for deflected } \\
\text { controls, and modified controls for } \\
\text { overset mesh }\end{array}$ & ULiv \\
\hline USAFA $[\underline{21}, \underline{23}]$ & Cobalt & Unstructured & SARC & Yes & $\begin{array}{l}\text { Additional tests using original F-17 } \\
\text { geometry modified controls for } \\
\text { overset mesh }\end{array}$ & $\begin{array}{l}\text { USAFA-Cobalt- } \\
\text { SARC }\end{array}$ \\
\hline USAFA $[\underline{21}, \underline{23}]$ & Cobalt & Unstructured & DDES-SARC & Yes & $\begin{array}{l}\text { Additional tests using original F-17 } \\
\text { geometry }\end{array}$ & $\begin{array}{l}\text { USAFA-Cobalt- } \\
\text { DDES-SARC }\end{array}$ \\
\hline USAFA [22] & Kestrel & Unstructured & SARC & Yes & $-\ldots$ & USAFA-Kestrel \\
\hline USAFA $[\overline{22}]$ & Kestrel & Unstructured & DDES-SARC & Yes & -- & $\begin{array}{l}\text { USAFA-Kestrel- } \\
\text { DDES }\end{array}$ \\
\hline
\end{tabular}

DLR $=$ German Aerospace Center, FOI = Swedish Defence Research Agency, NASA = National Aeronautics and Space Administration, USAFA = United States Air Force Academy, SA = Spalart Almaras, SARC = Spalart Almaras with Rotational Corrections, RSM = Reynolds Stress Model, EARSM = Explicit Algebraic Reynolds Stress Model, DDES $=$ Delayed Detached Eddy Simulation, SST $=$ Shear-stress transport. 


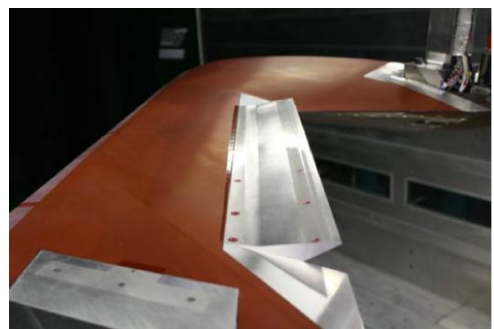

a) Photograph of detail of starboard control surfaces; image courtesy of DLR

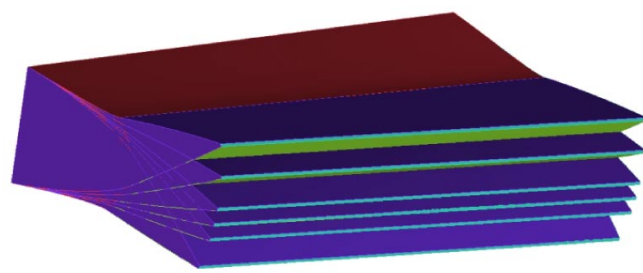

b) Set of inner flaps from original DLR-F17 geometry

Fig. 3 Set of new flaps derived from original DLR geometry.

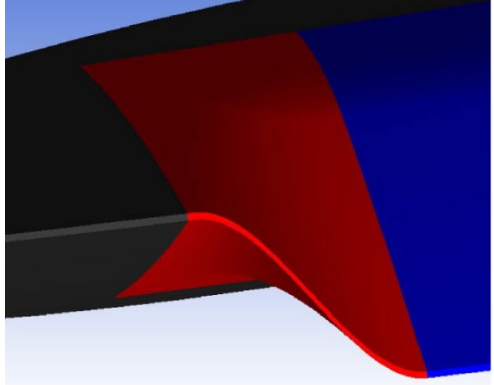

a) University of Liverpool modification [18]

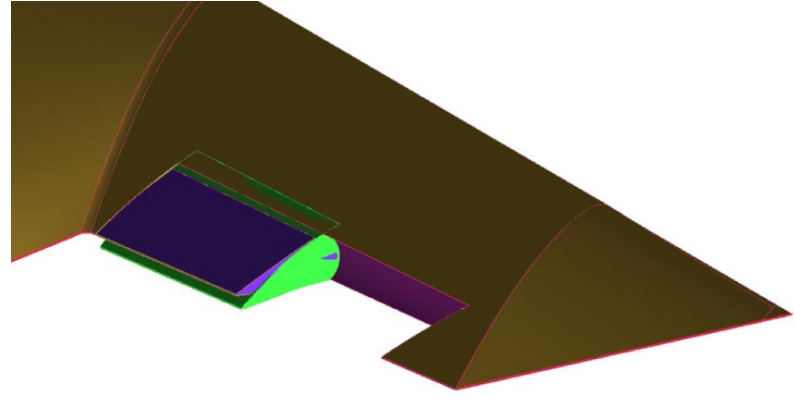

b) USAFA modification - overlay of original and modified geometry [21]

Fig. 4 Modification of the deflected control devices due to specific needs of participating organizations.

freestream velocity is $V=80 \mathrm{~ms}(263 \mathrm{ft} / \mathrm{s})$ in the closed test section and $V=70 \mathrm{~ms}(230 \mathrm{ft} / \mathrm{s})$ in the open test section. The model support system in the DNW-NWB includes basic support, halfmodel support, support for two-dimensional models, a rotary motion support for rolling and spinning tests, and a model positioning mechanism.

The model is the same model as used previously in the NATO RTO AVT-161 Task Group. In addition, it is equipped with trailing-edge control surfaces for tests of the control effectiveness for use in developing stability and control models. Figure 2 shows a schematic of this geometry with control devices and the sting. The controls are represented by inboard and outboard segments. All experimental data from the DNW-NWB wind tunnel were conducted at a Mach number of $M=0.15$ and a Reynolds number of $R e=1.56 \times 10^{6}$ (based on the root chord of the configuration). Details of the wind-tunnel tests are contained in $[10,13]$.

Although all CFD results are for the DLR F-17 geometry, the geometric representations used in all cases are not exactly identical. All but one participant used the sting geometry in their CFD meshes. There are also differences in how each group modeled the deflected control surfaces, depending on the requirements of each code.

The original DLR F-17 wind-tunnel model is made with controls that are nonmovable; a separate segment was designed and manufactured for each deflection angle. Each deflection is a combination of the control surface translation and rotation, with a certain part of the control surface being deformed to create a smooth surface transition between the aircraft and the deflected portion of the control surface. For example, Fig. 3 shows the set of inner flaps designed for different values of positive and negative deflections. The gaps between the deflected control surface and the surface of the aircraft are also not modeled. Because of this, modeling the control surfaces requires a different mesh for each modeled geometry unless some other meshing approach is used.

Table 3 Common dynamic cases: pitch motion [10] (unless otherwise indicated, all angles are in units of degrees)

\begin{tabular}{lrrrrrll}
\hline \hline TN2445 & LOB & LIB & RIB & ROB & $\Theta_{0}$ & $\Delta \Theta$ & $f, \mathrm{~Hz}$ \\
\hline $2342-2350$ & 0 & 0 & 0 & 0 & 10 & 4,7 & 1 \\
$2351-2359$ & 0 & 0 & 0 & 0 & 10 & 4,7 & 2 \\
$2666-2674$ & -20 & -20 & 20 & 20 & 10 & 4,7 & 0.94 \\
$2675-2683$ & -20 & -20 & 20 & 20 & 10 & 4,7 & 1.88 \\
$2360-2368$ & 0 & 0 & 0 & 0 & 15 & 4,7 & 1 \\
$2369-2377$ & 0 & 0 & 0 & 0 & 15 & 4,7 & 2 \\
$2648-2656$ & -20 & -20 & 20 & 20 & 15 & 4,7 & 0.94 \\
$2657-2665$ & -20 & -20 & 20 & 20 & 15 & 4,7 & 1.88 \\
\hline \hline
\end{tabular}

Table 2 Common static test cases $(L=$ left, $R=$ right, $\mathrm{IB}=$ inboard, $\mathrm{OB}=$ outboard) [10]; all angles are in units of degrees

\begin{tabular}{lrrrccr}
\hline \hline TN2445 & LOB & LIB & RIB & ROB & $\alpha$ & $\beta$ \\
\hline RN1001 & 0 & 0 & 0 & 0 & 10,15 & 0 \\
RN1092 & 0 & -20 & 20 & 0 & 15 & 0 \\
RN1114 & -20 & 0 & 0 & 20 & 10,15 & 0 \\
RN1103 & -20 & -20 & 20 & 20 & 10,15 & 0 \\
RN1007 & 0 & 0 & 0 & 0 & 10 & 10 \\
RN1008 & 0 & 0 & 0 & 0 & 14 & 3 \\
RN1109 & -20 & -20 & 20 & 20 & 10 & 10 \\
RN1110 & -20 & -20 & 20 & 20 & 14 & 3 \\
\hline \hline
\end{tabular}

Table 4 Common dynamic cases: yaw motion [10]

\begin{tabular}{lrrrrrrl}
\hline \hline TN2445 & LOB & LIB & RIB & ROB & $\Psi_{0}$ & $\Delta \Psi$ & $f, \mathrm{~Hz}$ \\
\hline $2270-2278$ & 0 & 0 & 0 & 0 & 10 & 5 & 1 \\
$2279-2287$ & 0 & 0 & 0 & 0 & 10 & 5 & 2 \\
$2162-2170$ & -20 & -20 & 20 & 20 & 10 & 5 & 0.94 \\
$2171-2179$ & -20 & -20 & 20 & 20 & 10 & 5 & 1.88 \\
$2288-2296$ & 0 & 0 & 0 & 0 & 15 & 5 & 1 \\
$2297-2305$ & 0 & 0 & 0 & 0 & 15 & 5 & 2 \\
$2180-2188$ & -20 & -20 & 20 & 20 & 15 & 5 & 0.94 \\
$2189-2197$ & -20 & -20 & 20 & 20 & 15 & 5 & 1.88 \\
\hline \hline
\end{tabular}


Two groups chose to model the control surface deflections in different ways. The first was the University of Liverpool, which used a methodology in which the control surfaces were modeled by a solid wall blending between the flap and wing, and then they deformed the baseline computational mesh so that it conformed to the deformed geometry. The second approach was that used by the U.S. Air Force Academy (USAFA), which used overset meshes to deflect the control surfaces. The USAFA made a modified model that used a fixed geometry control surface turning around a hinge line. Figure $\underline{4}$ shows both the University of Liverpool approach as well as the modified geometry and overlay of the original DLR F-17 inner control surface used in the USAFA approach. Additional consequences of having movable controls are the gaps that formed between the control
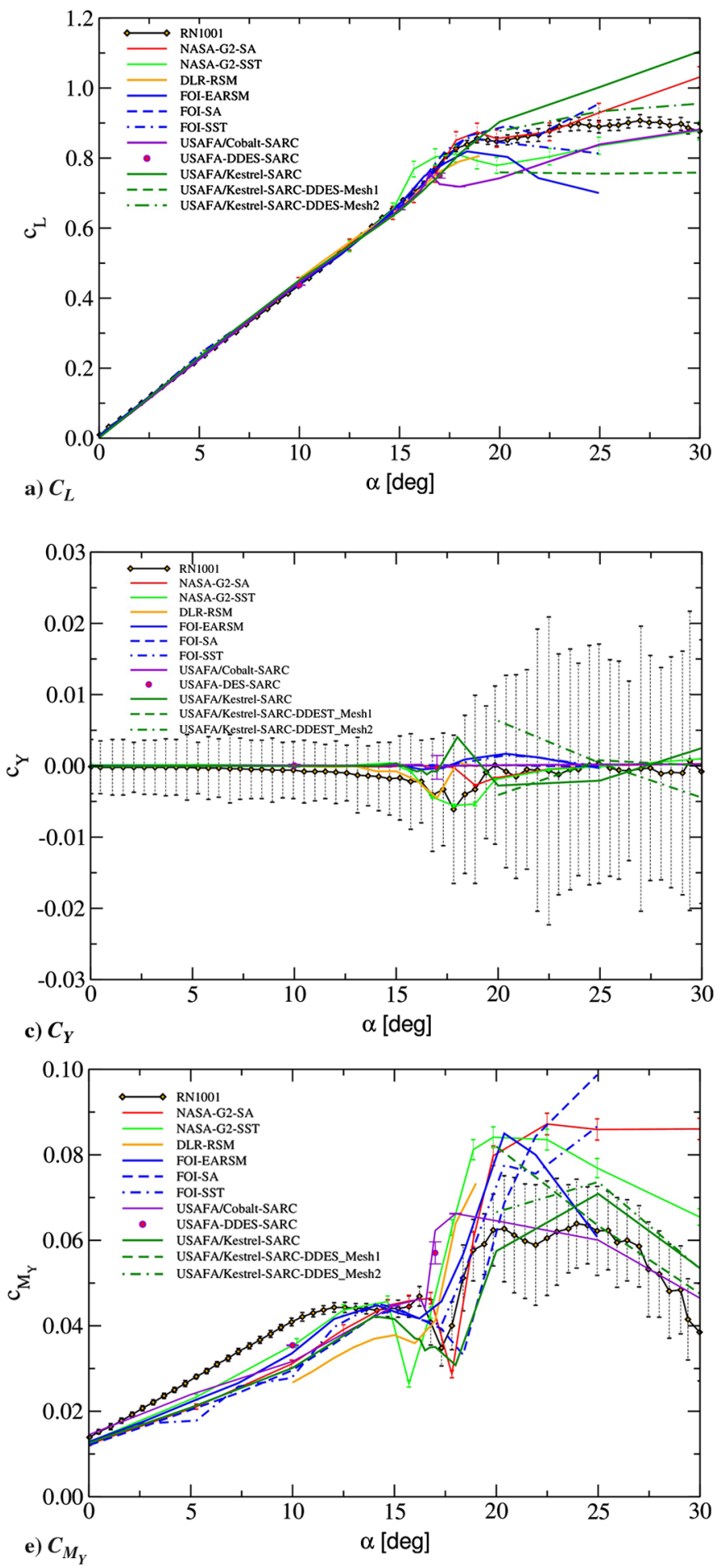

surface elements and the fixed surface of the aircraft. Due to the various approaches in modeling the control surfaces, these were the gaps along the control surface side edges and leading edges in slightly different ways by each group.

\section{Flow Cases}

The eight different cases were chosen as being mandatory for a static analysis: four symmetric flights at different angles of attack and deflections of control surfaces, and four nonsymmetric flight conditions at different sideslip angles. All results were for flight conditions with a Mach number of $M=0.15$ and a Reynolds number of $R e=1.56 \times 10^{6}$ (based on root chord), although
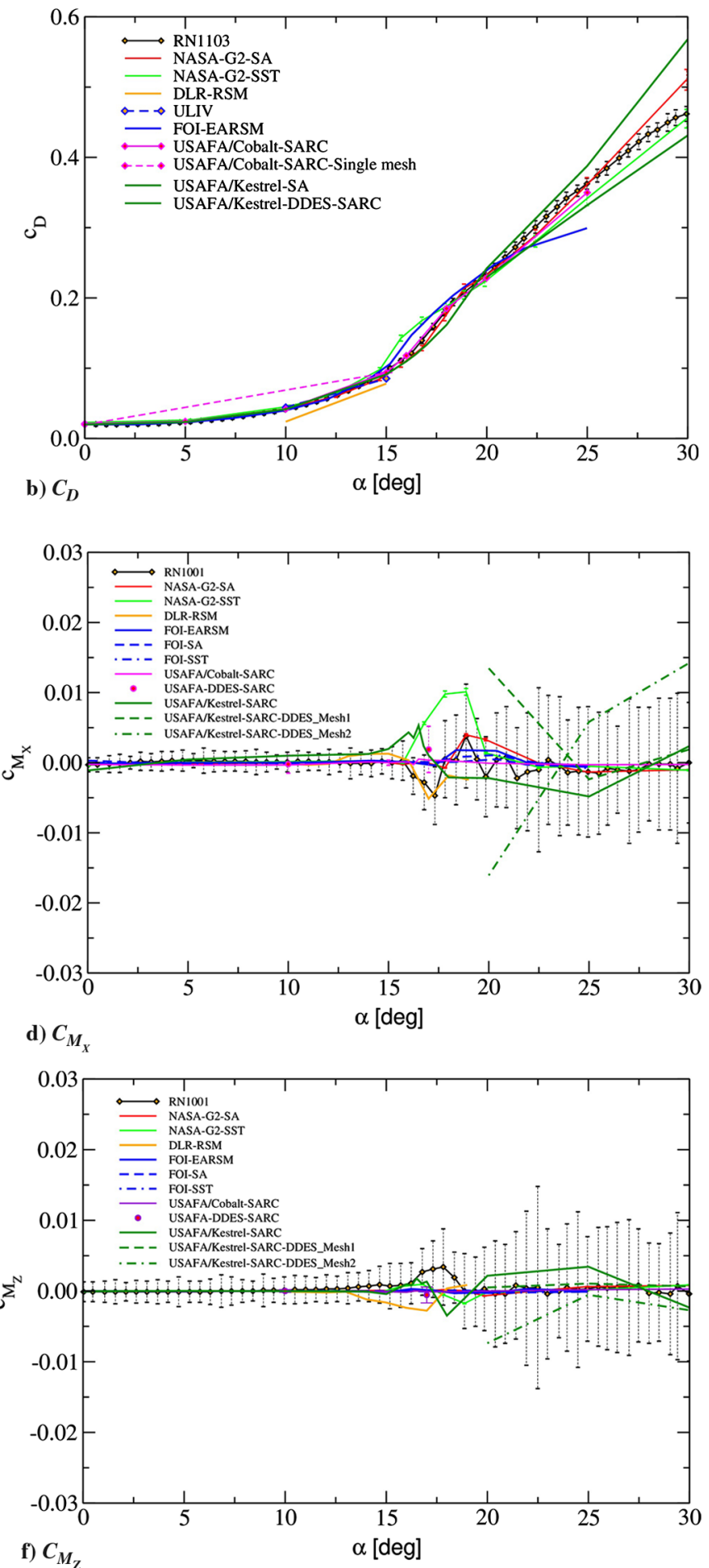

Fig. 5 Comparison of code predictions for static pitch case; RN1001 test case, $M=0.15, R e=1.56 \times 10^{6}$ (SA, Spalart-Almaras; RSM, Reynolds stress model). 

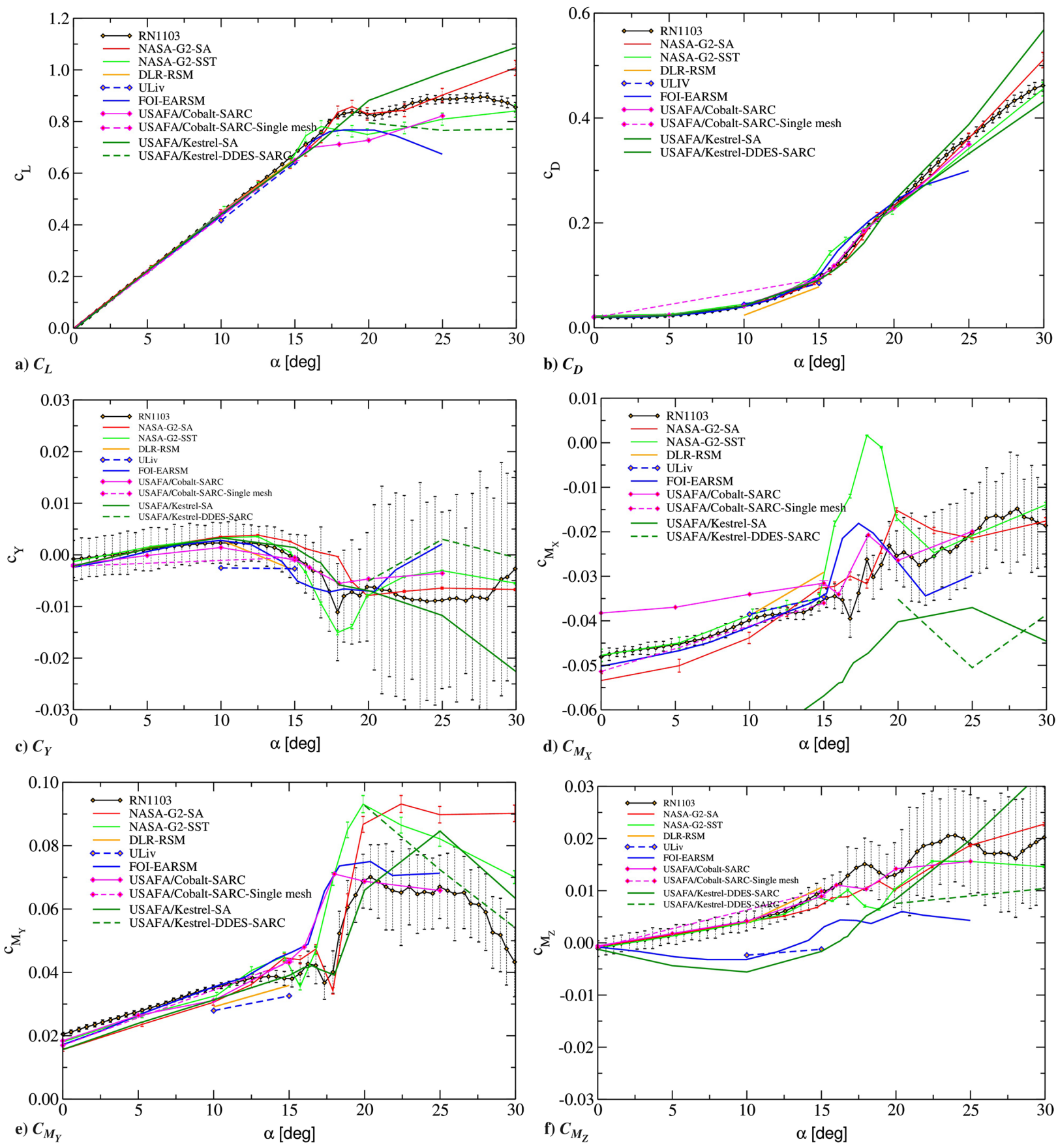

Fig. 6 Comparison of code predictions for static pitch case; RN1103 test case, $M=0.15, R e=1.56 \times 10^{6}$.

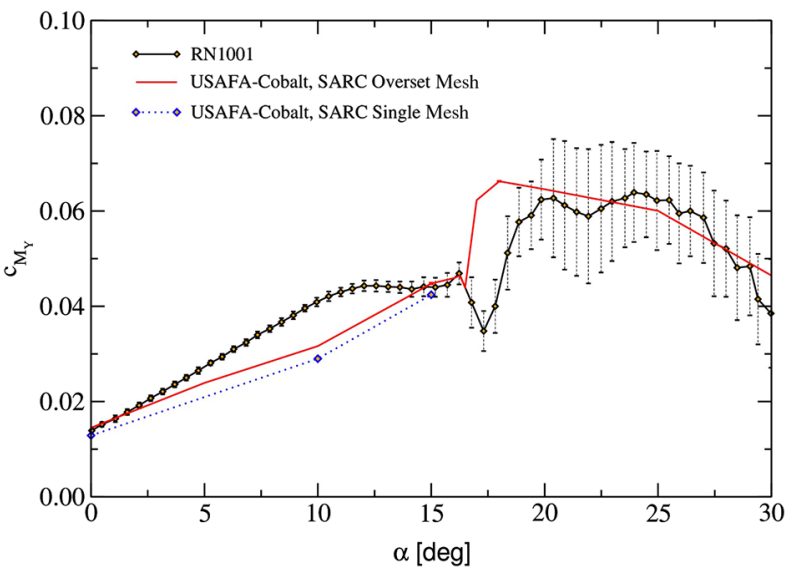

Fig. 7 Effect of modeling gaps in CFD mesh: RN1001 $M=0.15$, $R e=1.56 \times 10^{6}$. experiments were also conducted at transonic conditions with a smaller model and no control surfaces. Because of the asymmetric deflections of the controls, the test cases shown here were primarily interesting for roll control, although the comparisons were referred to for all forces and moments. Table 2 summarizes the static test cases shown in this paper; only results from runs 1001, 1103, 1007, 1008,1109 , and 1110 are presented and assessed.

The set of dynamic cases include pitch motion and yaw motion at different frequencies, as well as with and without deployed control surfaces. Tables $\underline{3}$ and $\underline{4}$ summarize the dynamic test cases.

\section{Results}

A total of five participating organizations submitted their available results for the common comparisons using six different CFD codes. The common case comparisons and assessment used a subset of selected cases that included both steady and time-dependent motion. Both the pitch and yaw motion had been considered to give a more 
complete picture of how well the CFD codes were predicting the aerodynamics of the vehicle.

\section{A. Static Cases}

The common static cases included four cases with various control deflections at various angles of attack, and the same cases were examined at a range of yaw angles. Two cases are shown for the static pitch motion and four cases are shown for static yaw motion.

\section{Pitch Variation}

The static pitch cases are tested and analyzed in a range of angles of incidence from 0 to $30 \mathrm{deg}$. The major characteristics of the

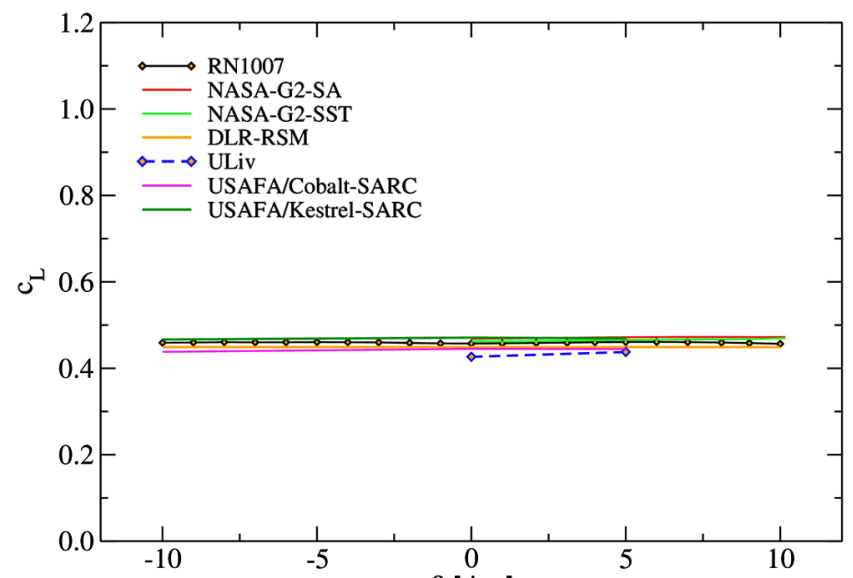

a) $C_{L}$

$\beta$ [deg]

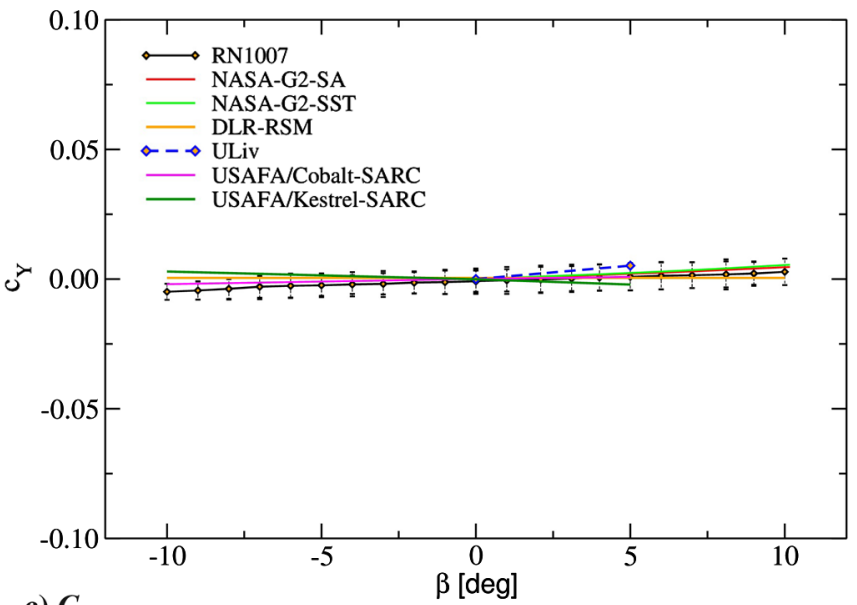

c) $C_{Y}$

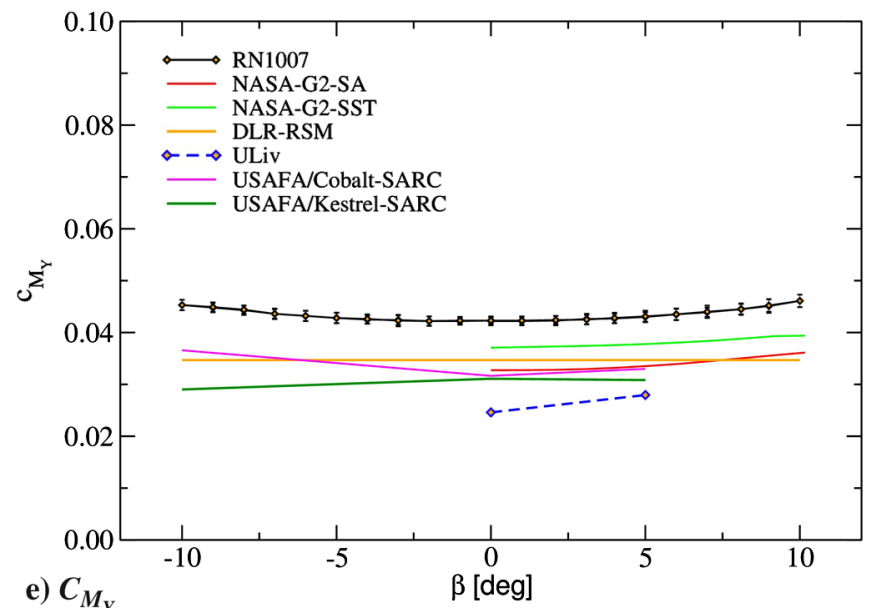

vortex structure above the unmanned combat aerial vehicle change dramatically with angle of attack, leading to several angle-ofattack ranges with unique characteristics [32]. In the range of angles of attack between 12 and $16 \mathrm{deg}$, the flow contains three separate vortices: the apex vortex, the thickness vortex, and the tip vortex. At approximately $16 \mathrm{deg}$ and higher, the tip vortex moves upstream and merges with the thickness vortex. This character of the flowfield lasts up to angles of attack larger than $18 \mathrm{deg}$ when the tip-thickness vortex merges with the apex vortex and the flowfield above the wing consists of only one vortex, which then brakes down at angles of attack above $20 \mathrm{deg}$. The flow is entirely attached at angles below $10 \mathrm{deg}$; at higher angles, the flow starts to separate
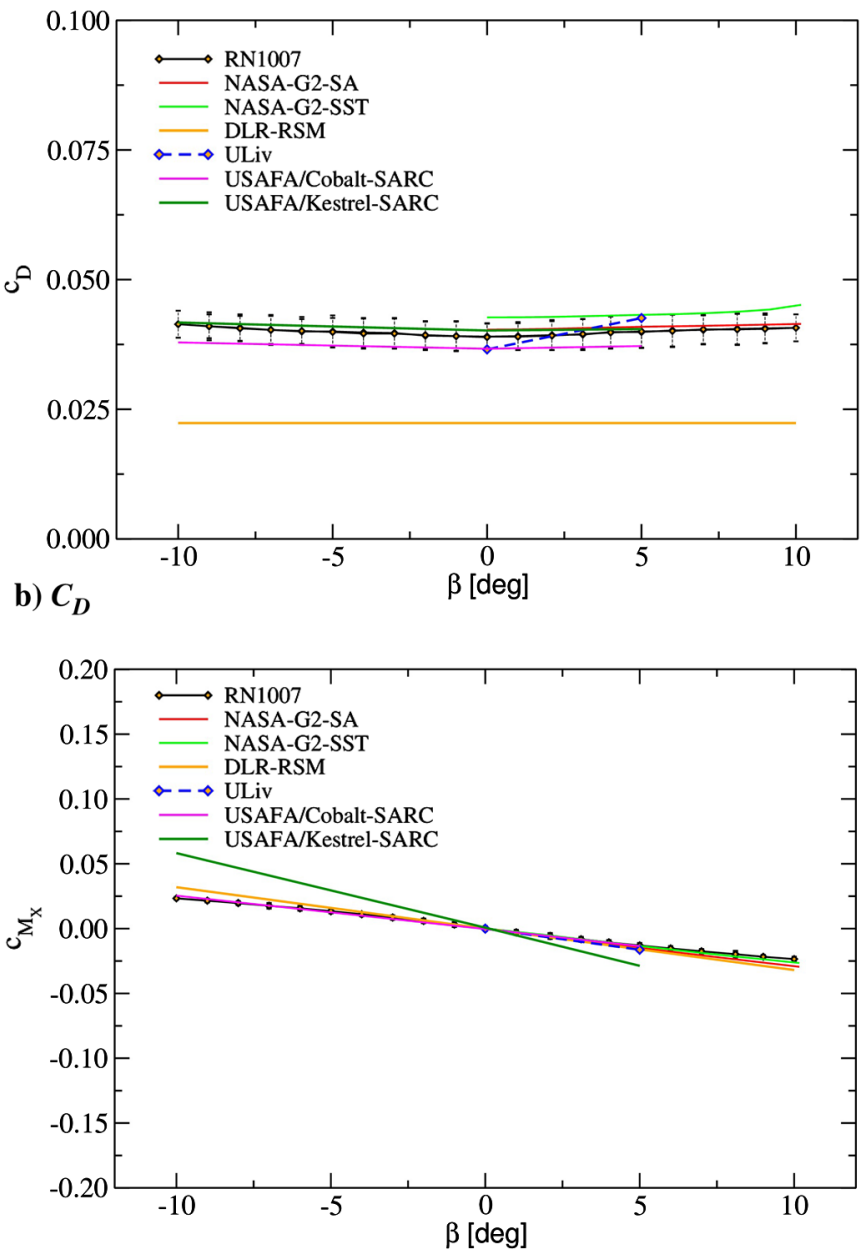

d) $C_{M_{X}}$

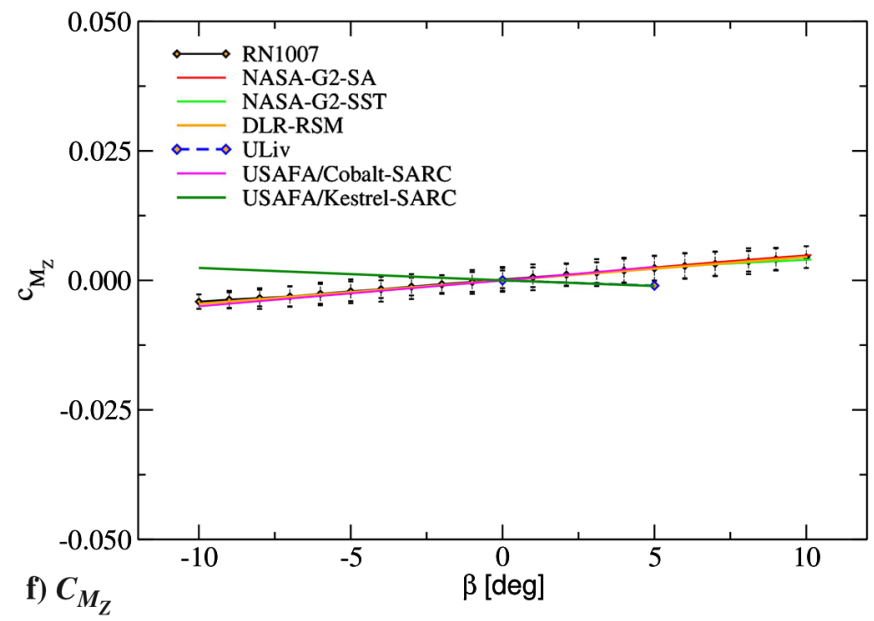

Fig. 8 Comparison of static sideslip predictions at an angle of attack of $10 \mathrm{deg}$; RN1007 test case, $M=0.15, R e=1.56 \times 10^{6}$. 
at the wingtip and the separation region progresses upstream as the angle of attack increases [32].

Figures $\underline{5}$ and $\underline{6}$ show the comparisons of the force and moment predictions from various codes with the available experimental data. The difference between the two cases is the deflection of the control surfaces, which is zero in the first case (RN1001) and \pm 20 deg in the second case (RN1103). The general characteristics of the flowfield are very similar for both cases, which is due to the relatively low effectiveness of the controls.

The lift and drag predictions show the best agreement with the wind-tunnel data. The major deviations begin to occur at angles of attack around $18 \mathrm{deg}$, which are where the flow transitions to one major vortex above the wing, as described previously.
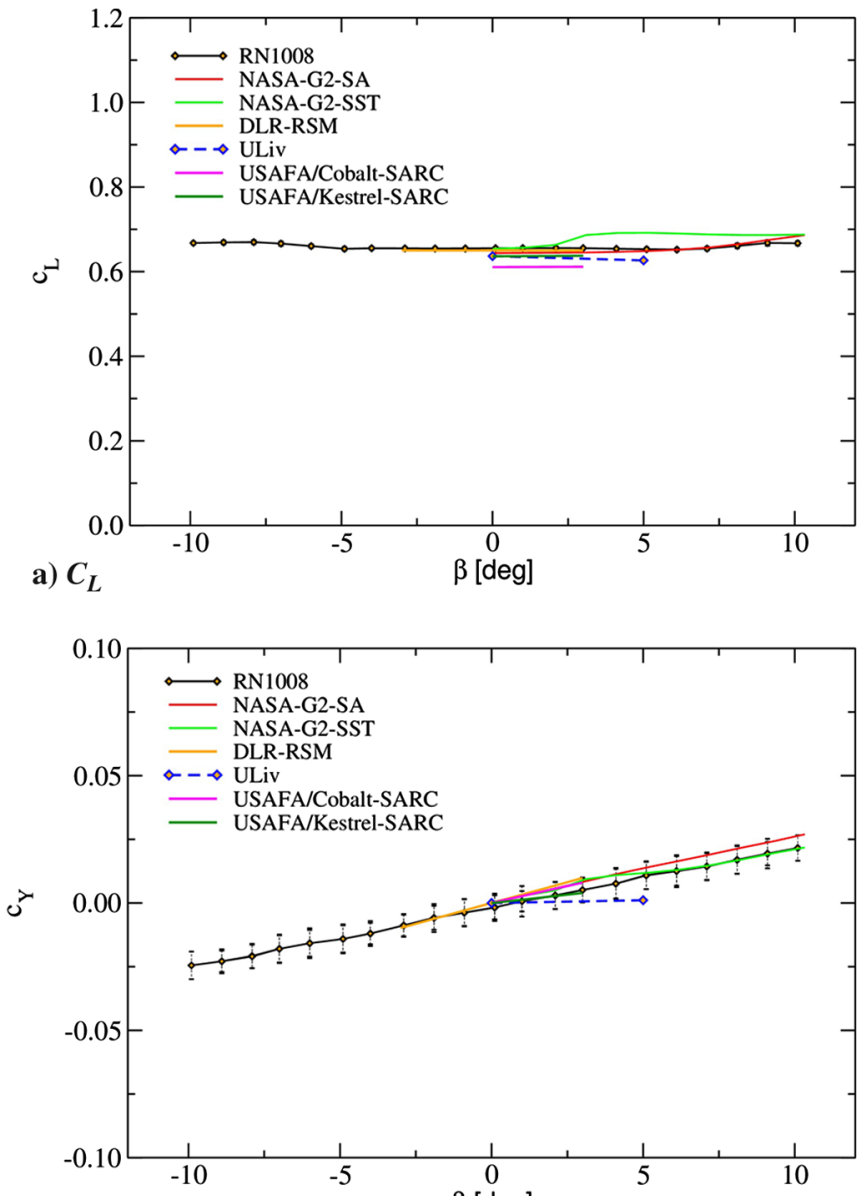

c) $C_{Y}$

$\beta[$ deg]

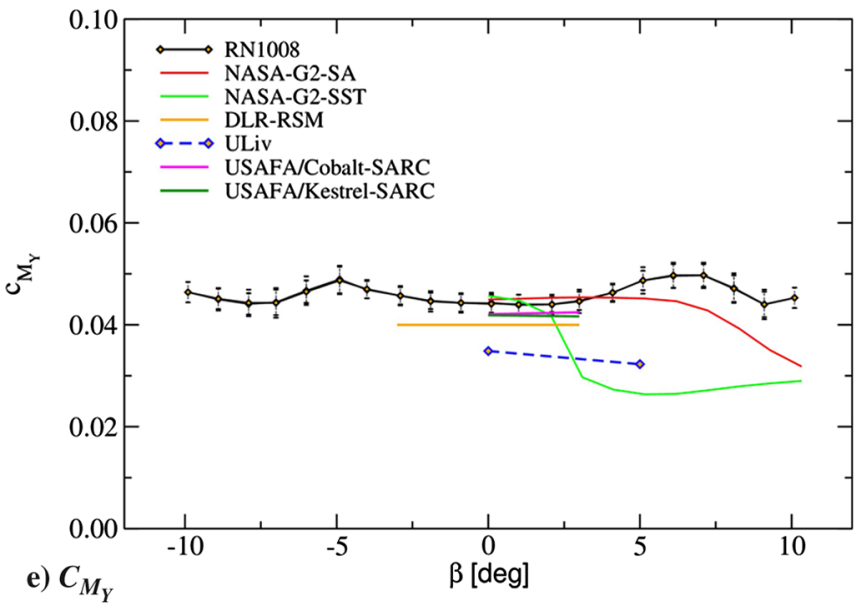

The pitch moment is predicted fairly well within the linear range, with all predictions being reasonably close to each other. The only exception is the prediction that does not consider the sting geometry in the CFD mesh and predicts lower values of the pitch moment (previous studies showed that the sting created an offset in pitching moment). All codes are able to predict the sudden rise of the pitching moment, which occurs at around $18 \mathrm{deg}$ in the wind-tunnel data. A few of the codes predict fairly well the extent of pitch moment variations, which occur at an angle of incidence of $15 \mathrm{deg}$ in the windtunnel data; other codes only predict a very small break. This is because the multiple vortices, and their interaction, are dominant in this angle-of-attack range, and their accurate prediction is strongly dependent on the turbulence model and grid density used. All codes
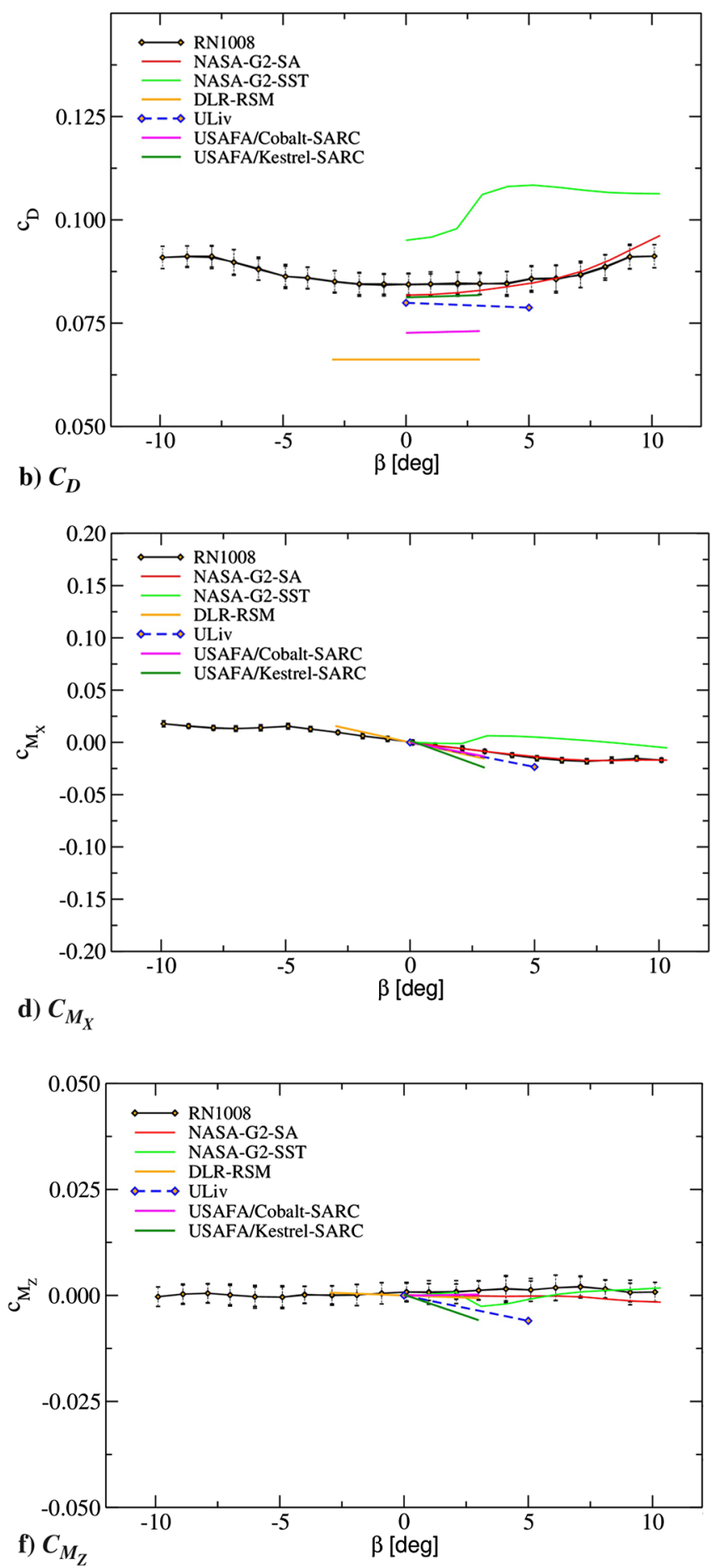

Fig. 9 Comparison of static sideslip predictions at an angle of attack of $10 \mathrm{deg}$; RN1008 test case, $M=0.15, R e=1.56 \times 10^{6}$. 
have problems in predicting the weak nonlinearity, which starts at an angle of incidence around $11 \mathrm{deg}$, which is very possibly a consequence of omitting the wind-tunnel walls from the CFD setup [34].

For the effect of gaps on the CFD solution, the USAFA simulation has used the overset mesh to model the control device deflections; as mentioned earlier, this approach requires some changes to the original shape of the controls. In addition, this approach requires modeling the gaps between each control surface and the wing. Tests were done to determine to what extent the modeling of gaps in the CFD mesh affected the solution. The differences between the
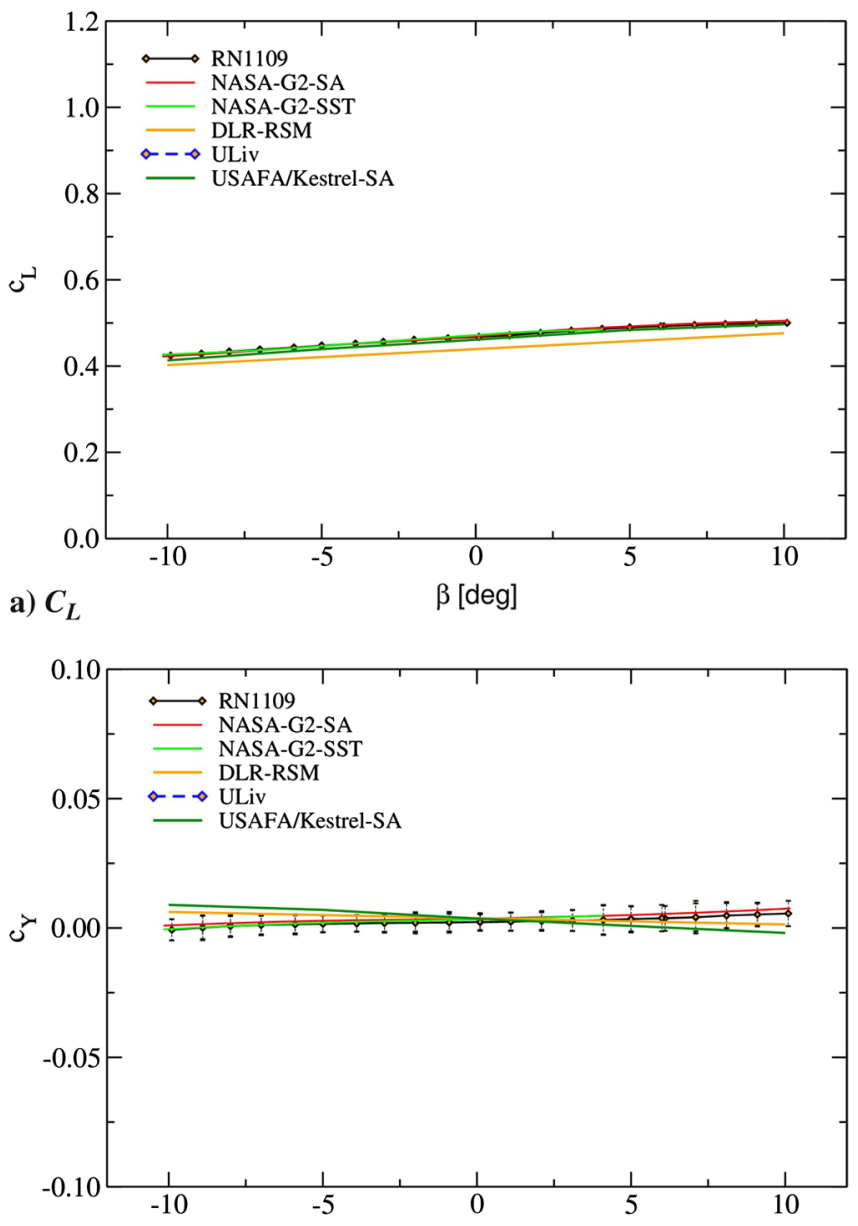

c) $C_{Y}$

$\beta$ [deg]

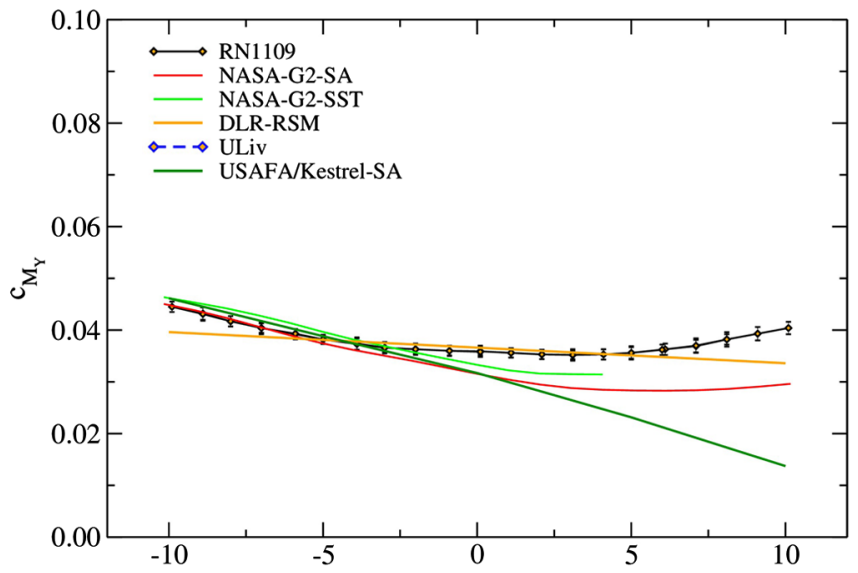

e) $C_{M_{Y}}$

$\beta$ [deg] solutions with and without modeled gaps are about $\Delta C_{L} \approx 0.01$ in lift and about $\Delta C_{D} \approx 15$ drag counts in drag. Figure 7 shows the effect that modeling the gaps has on the pitching moment, which causes a constant offset in the results. It should be noticed that the first mesh that models gaps is an overset mesh, and the second without gaps is a single mesh. The surface resolution is identical for both meshes.

\section{Yaw Variation}

The yaw variation was not studied at all during the previous NATO RTO Task Group AVT-161. However, the yaw cases have been included as one of the mandatory common cases for AVT-201.
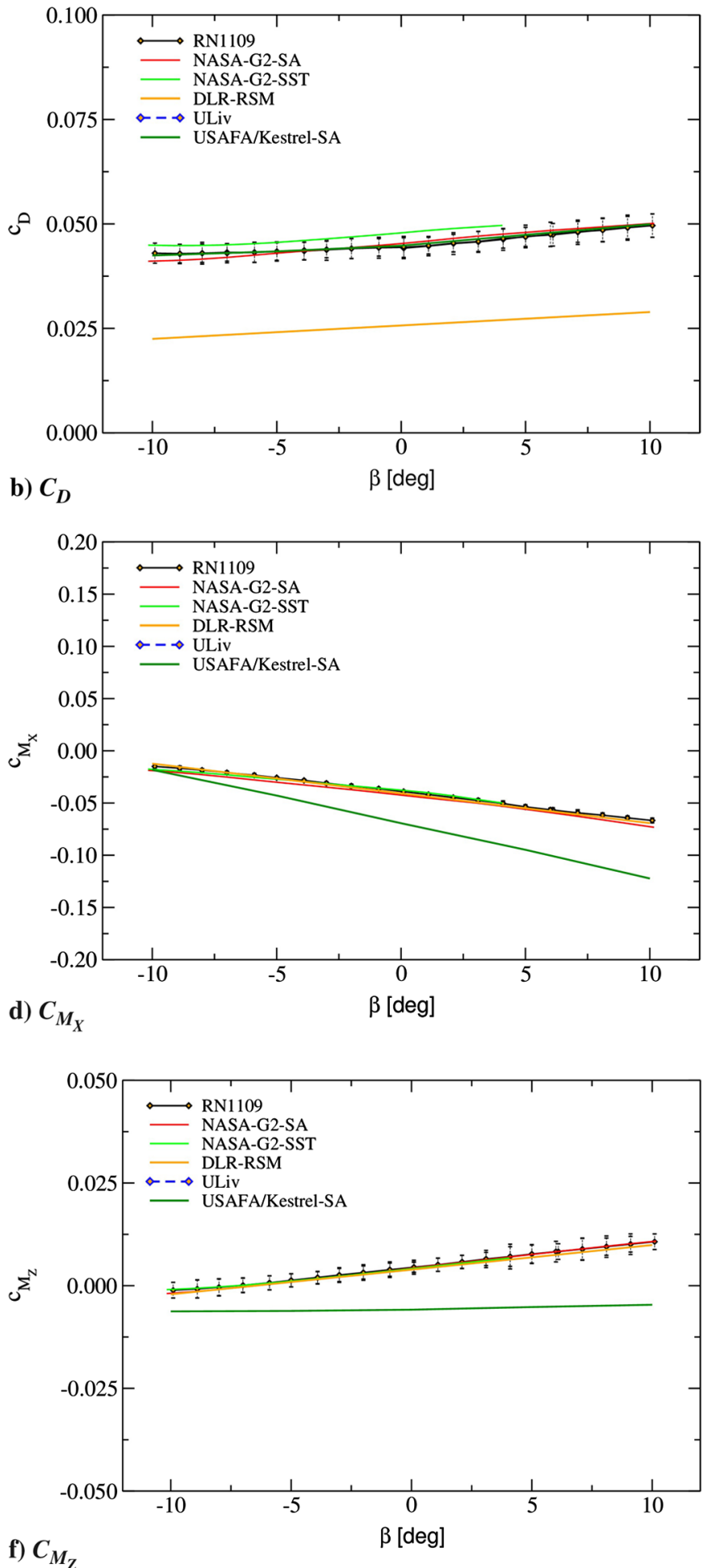

Fig. 10 Comparison of static sideslip predictions at an angle of attack of $10 \mathrm{deg}$; left control surfaces at -20 deg, right control surfaces at +20 deg; RN1109, $M=0.15, R e=1.56 \times 10^{6}$. 
Unlike the pitch variation, which has been studied extensively (leading to a relatively good understanding of the flow around this aircraft at different angles of incidence [32]), the yaw motion has not attracted as much interest.

The static yaw cases tested are at two angles of incidence: $\alpha=10 \mathrm{deg}$ and $\alpha=14 \mathrm{deg}$. This is still within the region of linear and weakly nonlinear behavior of the configuration. The sideslip flow characteristics are linear in the range of tested yaw angles.

All four common cases are shown in Figs. $\underline{8}-11$; the differences between the cases with control deflections and without control deflections are relatively small, and it does not appear that the control deflections substantially change the character of the flow for these cases. This is due to the spanwise flow over the upper surface of the
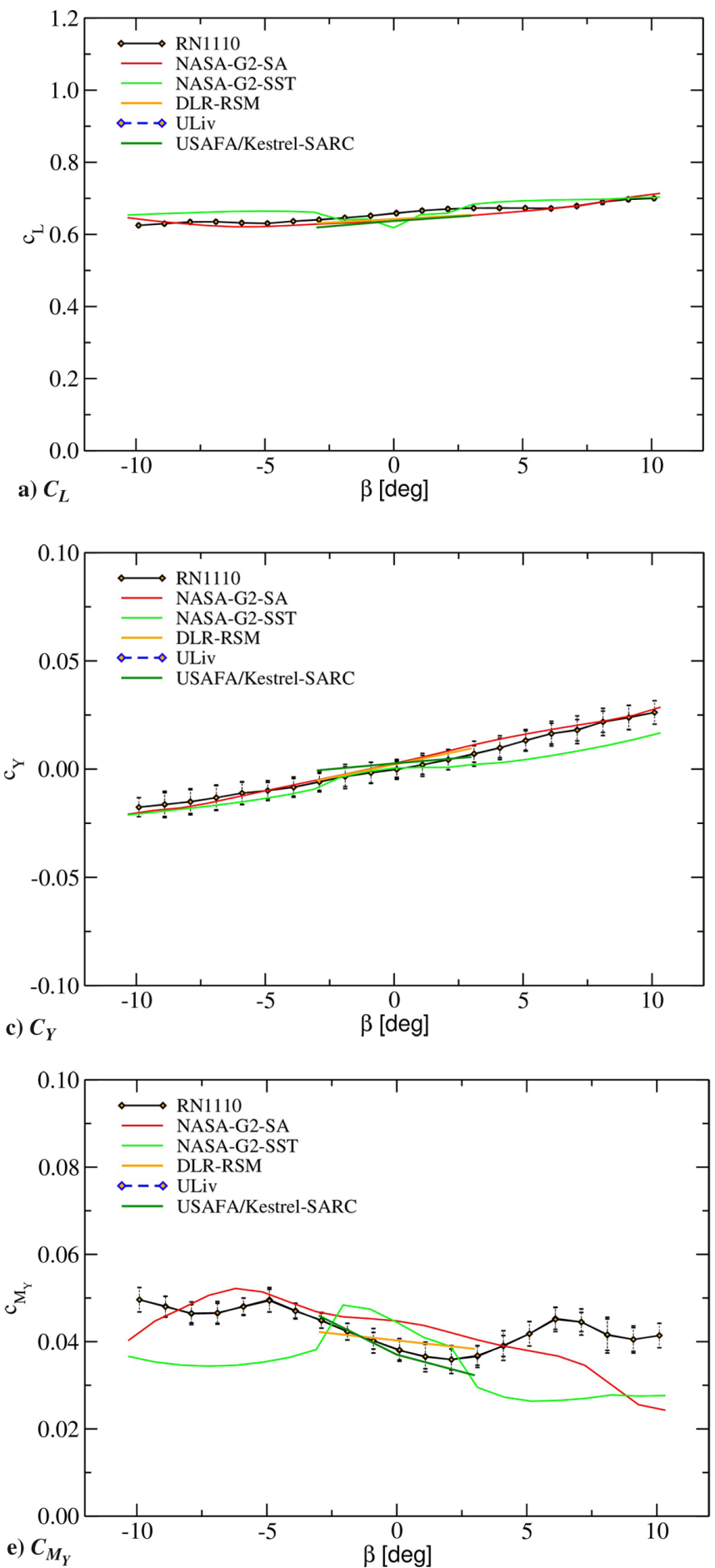

wing aligning with the control surface angle, leading to very little flow directly over the control surfaces. This will be discussed at greater length in the next section.

\section{B. Effectiveness of Control Surfaces}

The effectiveness of controls surfaces is generally lower on these types of aircraft configurations because the flow tends to align itself with the direction of the trailing edge at low angles of incidence, effectively negating any effect of surface curvature caused by flap deflections.

Figure 12 shows the comparisons of the control effectiveness for the aircraft at different angles of incidence and a zero angle of sideslip. The two cases that are used to measure effectiveness are

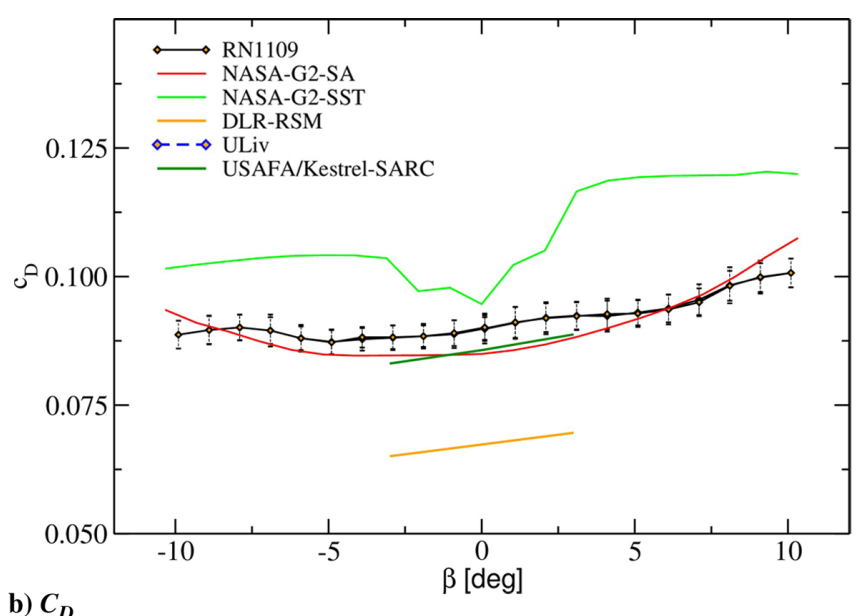

b) $C_{D}$
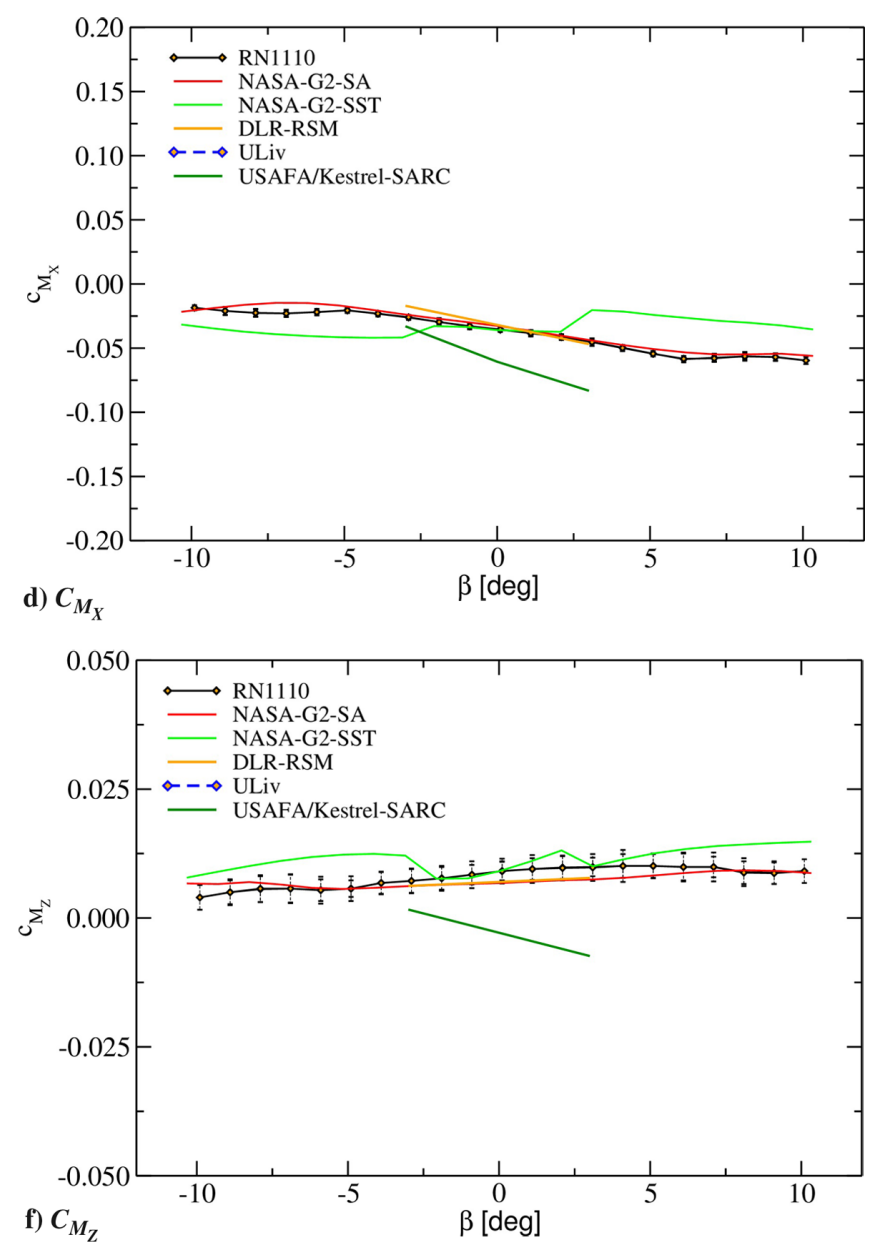

Fig. 11 Comparison of static sideslip predictions at an angle of attack of $14 \mathrm{deg}$; left control surfaces at -20 deg, right control surfaces at +20 deg; RN1110, $M=0.15, R e=1.56 \times 10^{6}$. 

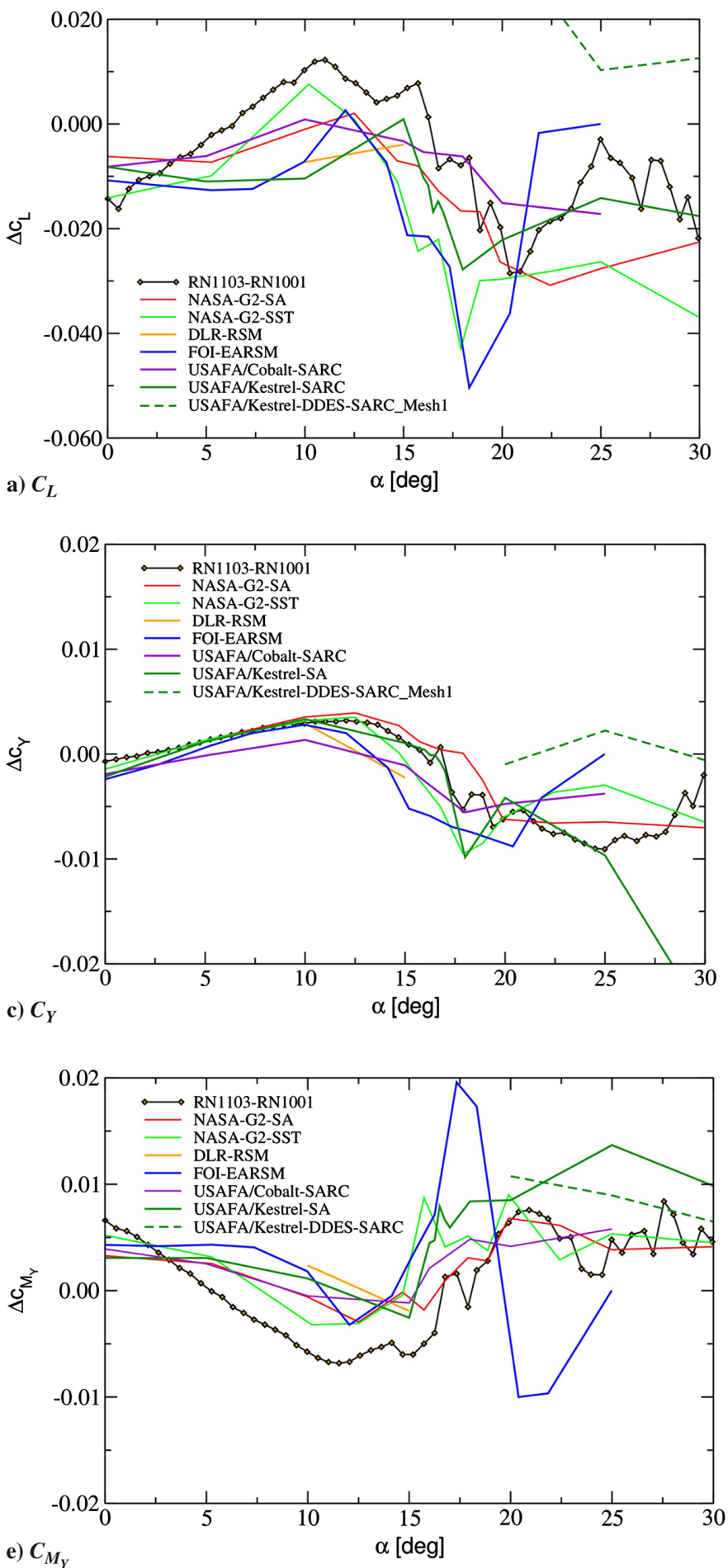

e) $C_{M_{Y}}$

$\alpha$ [deg]

Fig. 12 Comparison of incremental coefficie
RN1103-RN1001, $M=0.15, R e=1.56 \times 10^{6}$.

RN1103 (all left controls deflected at $-20 \mathrm{deg}$, and all right controls deflected at $+20 \mathrm{deg}$ ) and RN1001, which is a case with no control deflections. The corresponding data for the two cases are shown in Figs. 5 and 6 . The effect of the control surface deflections on the lift and pitch moment coefficients is very small, which is due to the combination of low control effectiveness for these types of aircraft (as mentioned previously) and by the antisymmetric deflection of the controls. The effect of the deflections on the roll and yaw moment coefficients is much stronger, as would be expected for an equivalent aileron deflection. The trend of the moment curves is predicted generally well at the lower angles of incidence, however, the accuracy of the predictions is reduced at the higher angles of incidence where the flow is strongly nonlinear. There is a larger difference in
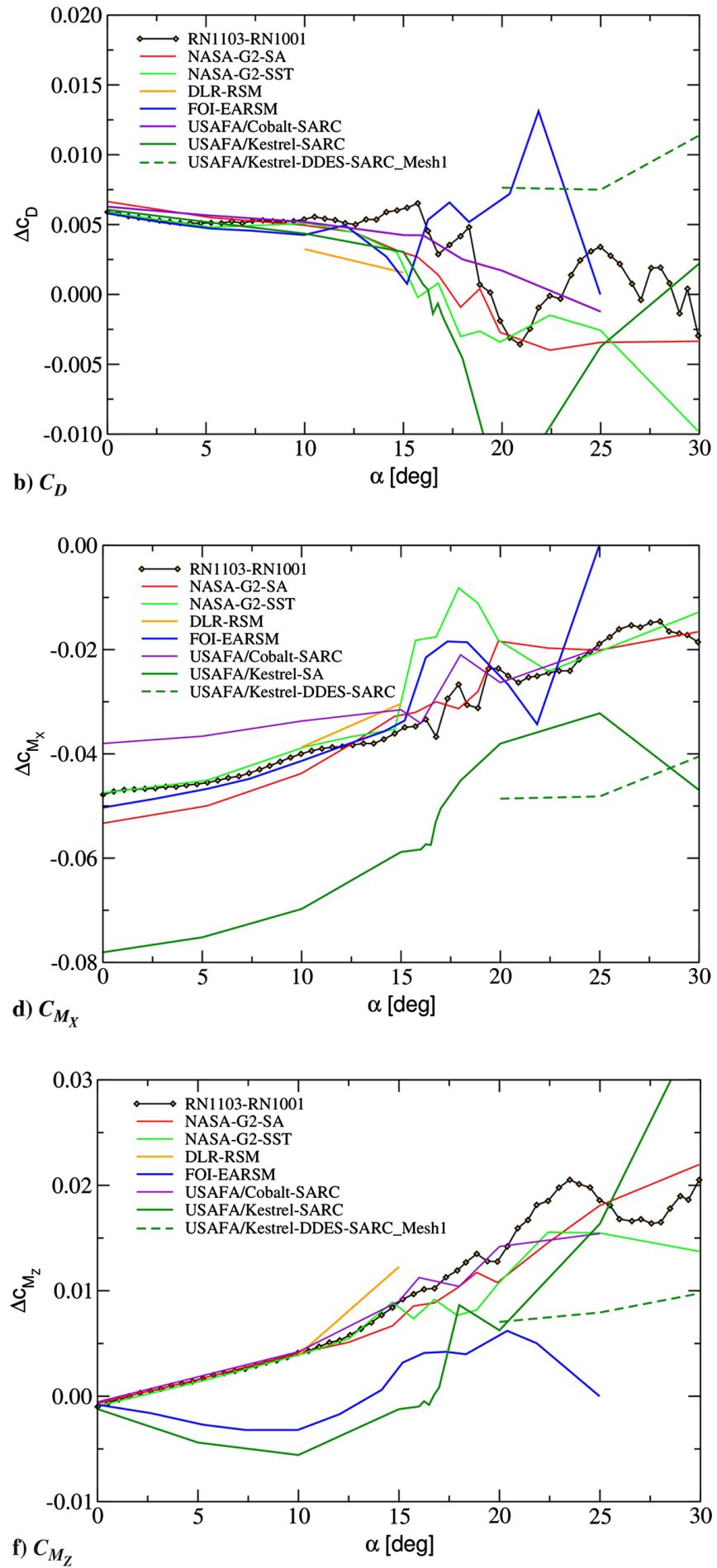


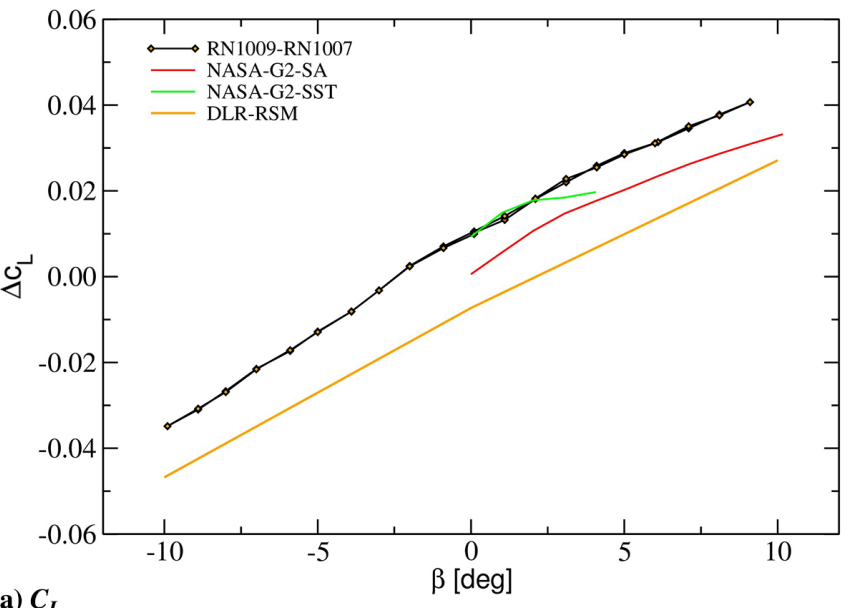

a) $C_{L}$

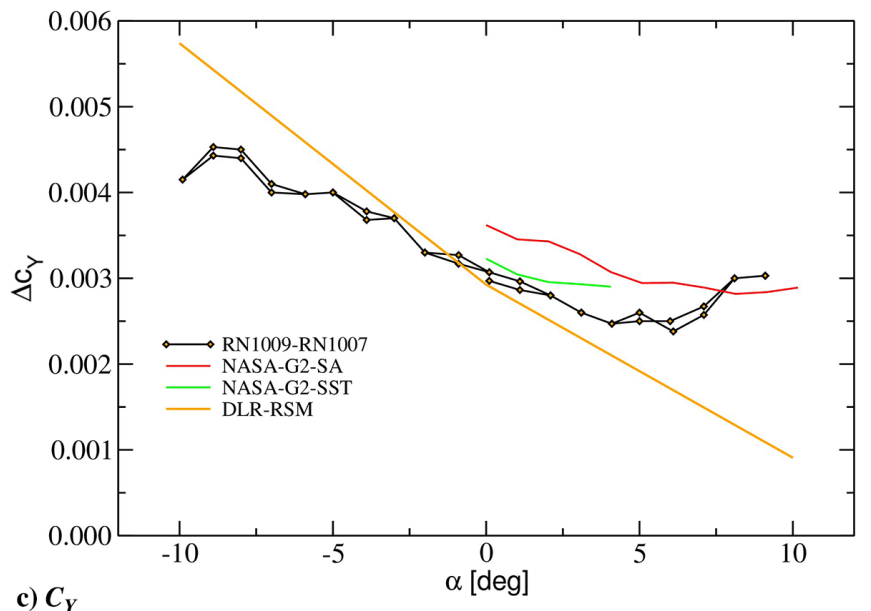

c) $C_{Y}$

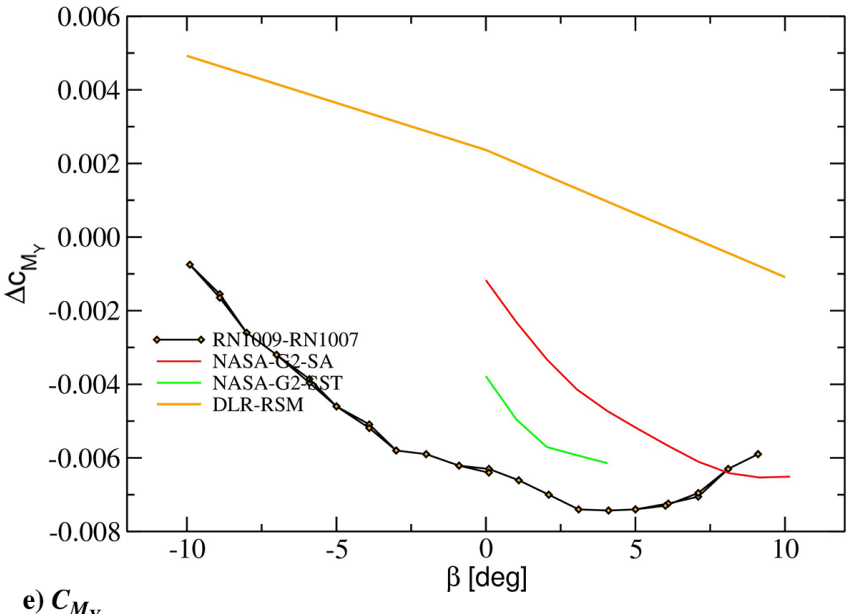

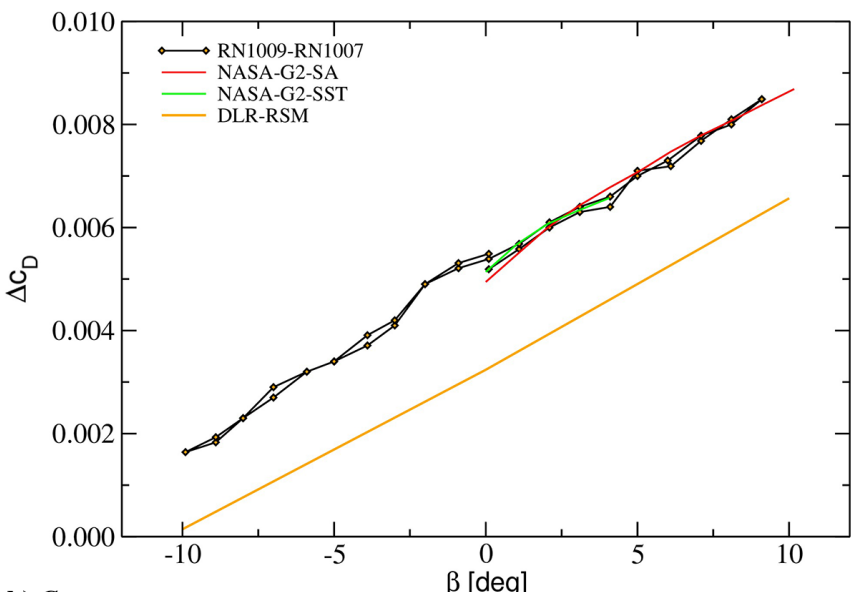

b) $C_{D}$
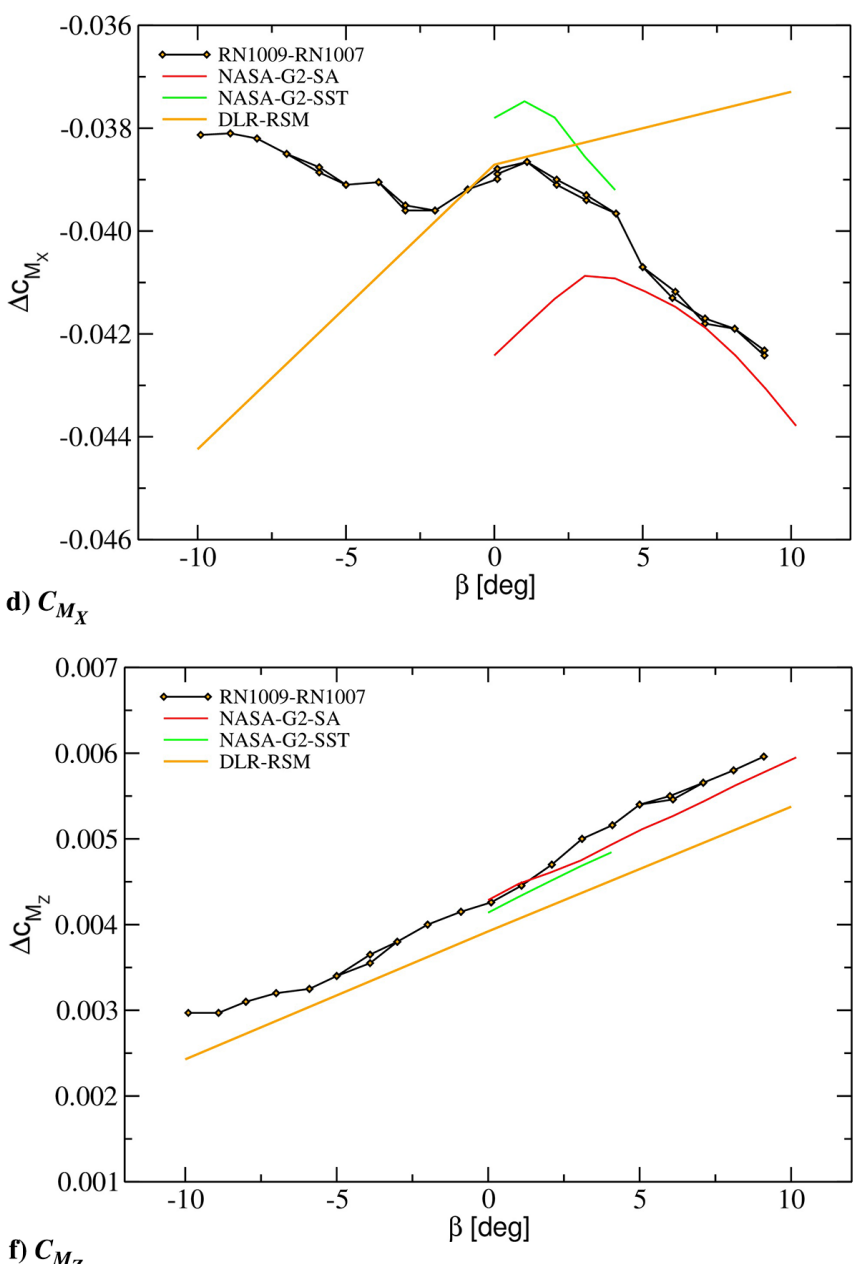

Fig. 13 Comparison of incremental static sideslip predictions at an angle of attack of $10 \mathrm{deg}$; left control surfaces at -20 deg and right control surfaces at +20 deg; RN1009-RN1007, $M=0.15, R e=1.56 \times 10^{6}$.

moment coefficient at higher yaw angles, which is generally less well predicted. It should be noted that the angles of incidence for the static yaw common cases take place before the strong nonlinearity in the pitch moment occurs.

\section{Dynamic Case}

\section{Pitch Motion}

The first dynamic motion considered is a sinusoidal pitch motion around the angle of incidence of $10 \mathrm{deg}$ with an amplitude of $4.7 \mathrm{deg}$ at a frequency of $1 \mathrm{~Hz}$. Figure 15 shows the computationally obtained forces and moments compare to wind-tunnel data. The pitch motion occurs in the range of angles of attack where the lift is linear; the pitch moment begins to behave weakly nonlinearly at an angle of attack of approximately $12 \mathrm{deg}$.

The two predictions included in the comparisons come from National Aeronautics and Space Administration (NASA) and the University of Liverpool, and only the last cycle of each prediction is plotted. In both cases, the predictions of the axial force $C_{x}$ is good; the prediction of the normal force is somewhat less accurate, with the largest differences of about 8 and 10\%, respectively.

An interesting result is the comparison of the pitch and roll moments. Both CFD results predict lower values of the pitch moment at lower angles of incidence. This cannot be attributed to the effect of the sting because both results consider the sting geometry in their 


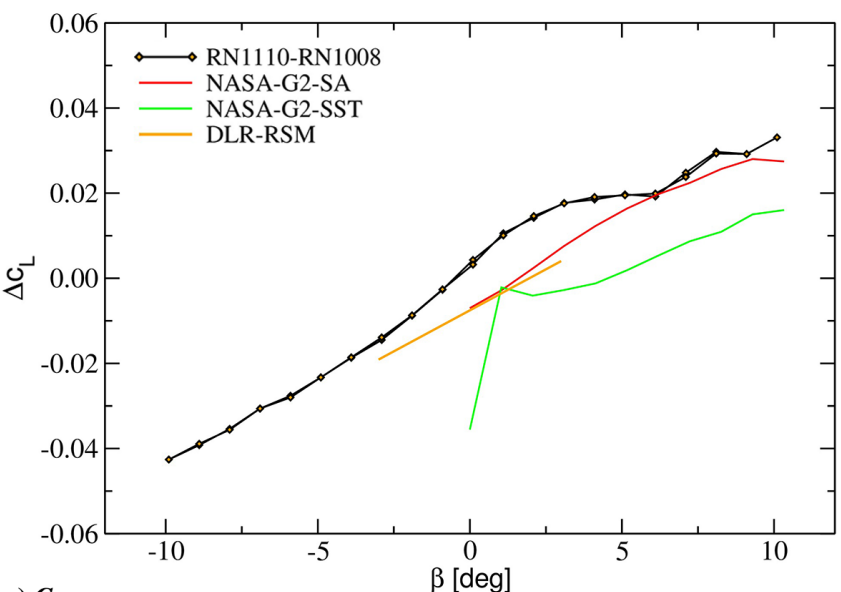

a) $C_{L}$

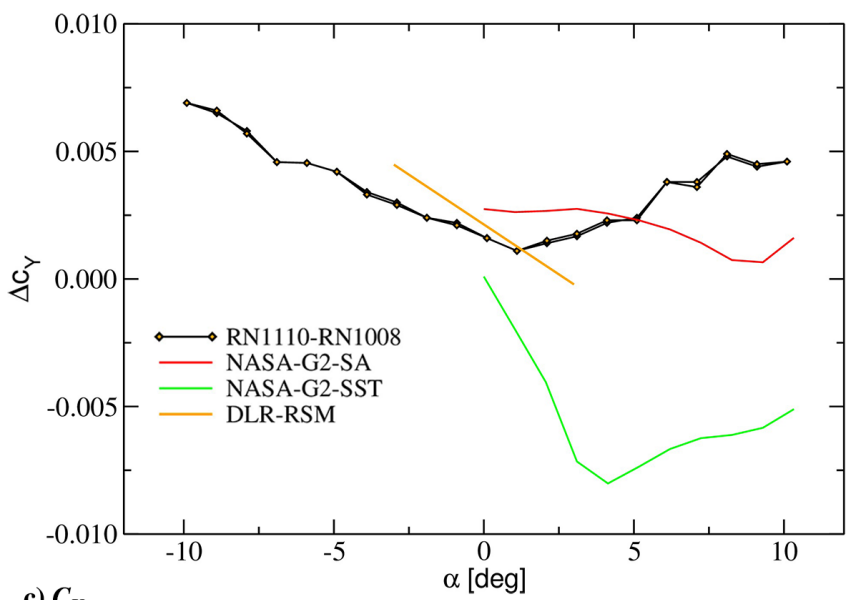

c) $C_{Y}$

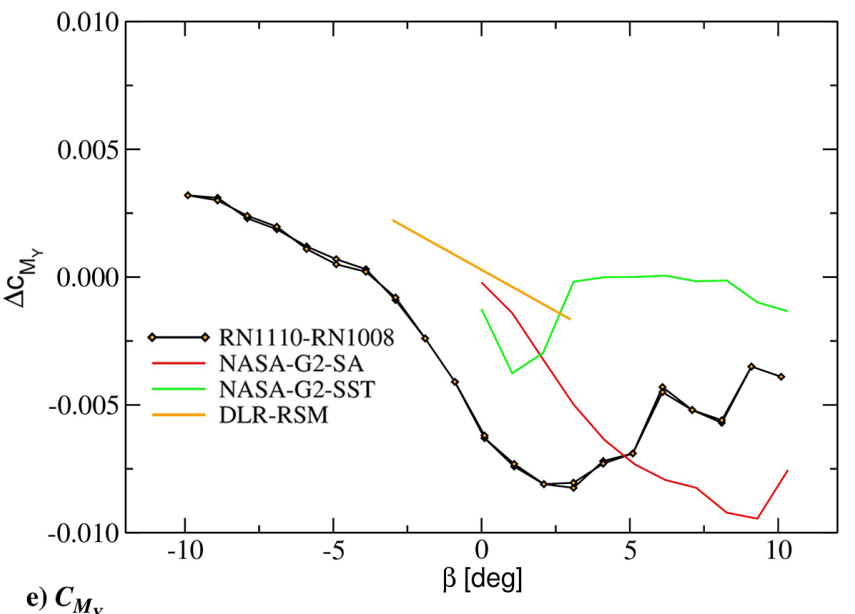

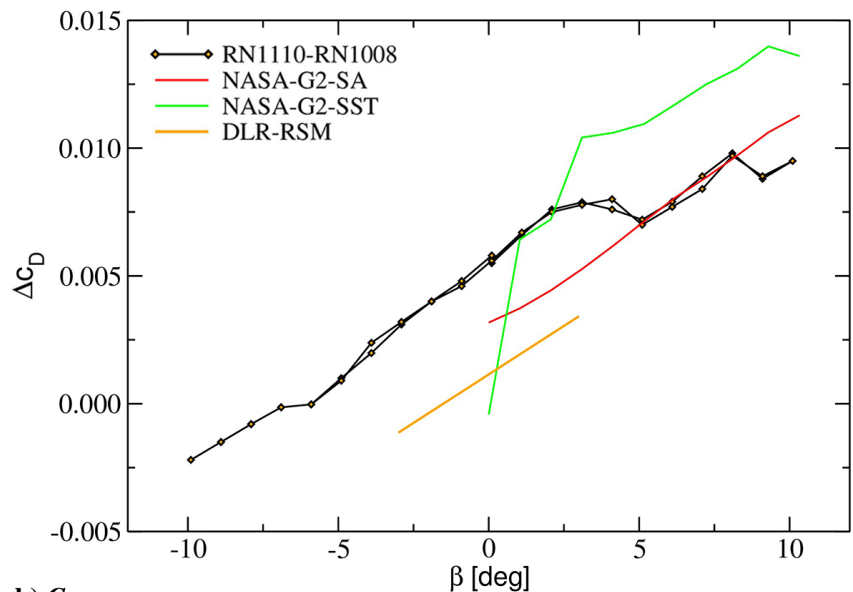

b) $C_{D}$
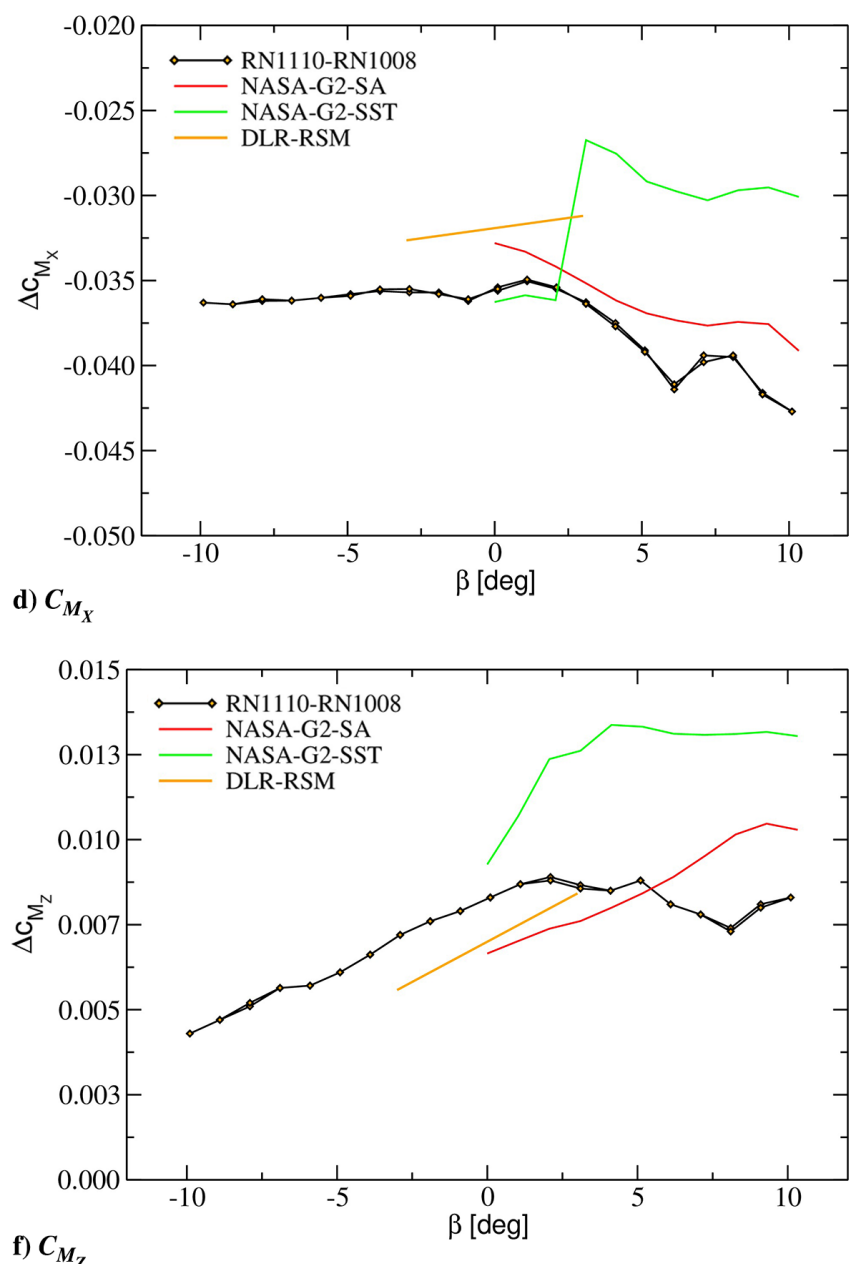

Fig. 14 Comparison of incremental static sideslip predictions at an angle of attack of $10 \mathrm{deg}$; left control surfaces at -20 deg, and right control surfaces at +20 deg; RN1110-RN1008, $M=0.15, R e=1.56 \times 10^{6}$.

CFD meshes. None of the CFD results are able to reproduce the nonlinearity in pitch moment to an extent shown by the wind-tunnel data, although the NASA results show an indication of a possible nonlinearity at around an angle of incidence of $14 \mathrm{deg}$.

2. Yaw Motion

The second common case for dynamic motion shown in this paper is the case of yaw motion. The motion is a sinusoidal motion around a sideslip angle of 0 deg with an amplitude of $5 \mathrm{deg}$ and a frequency of $1 \mathrm{~Hz}$. The comparisons here are generally better than the pitch motion case, which is probably due to the linear character of the flow around the aircraft at these conditions; see Fig. 16.

\section{Conclusions}

The paper summarizes the analysis of the results of five participating organizations and six different numerical codes. Five of the codes use unstructured meshes, and one code uses structured meshes (the Liverpool code). The details of the codes as well as computational meshes are not described in great detail here, but each approach is detailed in the papers referenced in Table 1. This is intentional because the main purpose of this paper is not to look at details of the different numerical codes but, rather, to consider the numerical codes as a tool available to a group of researchers for the study of the strongly nonlinear aerodynamic characteristics of the SACCON aircraft, and an 


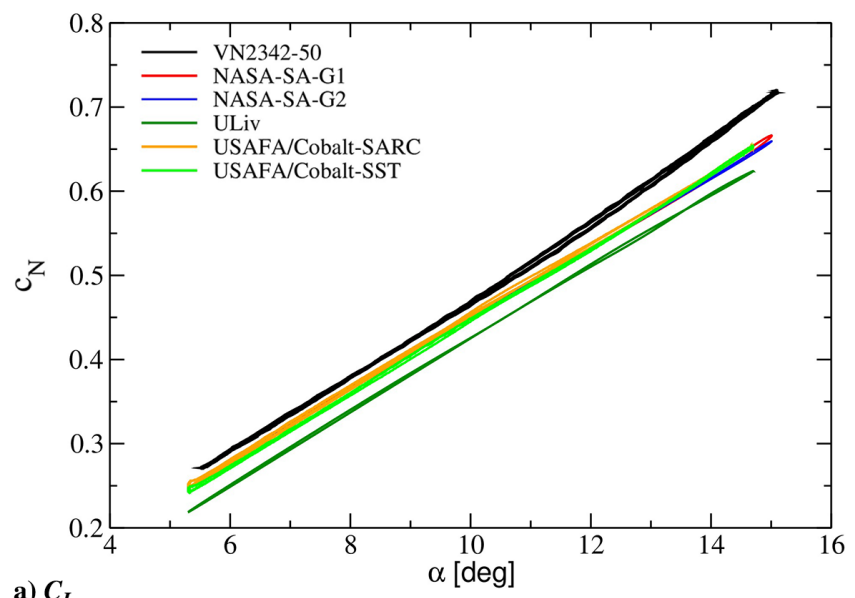

a) $C_{L}$

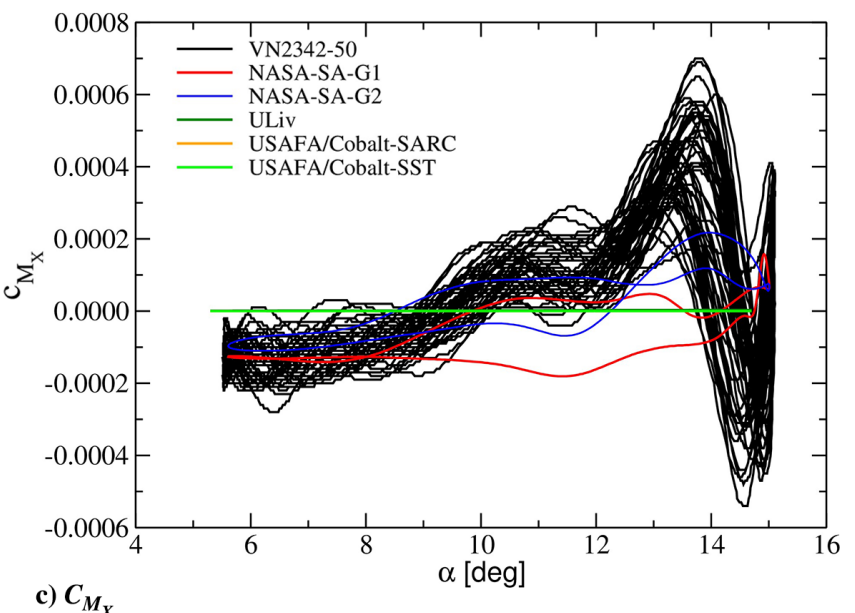

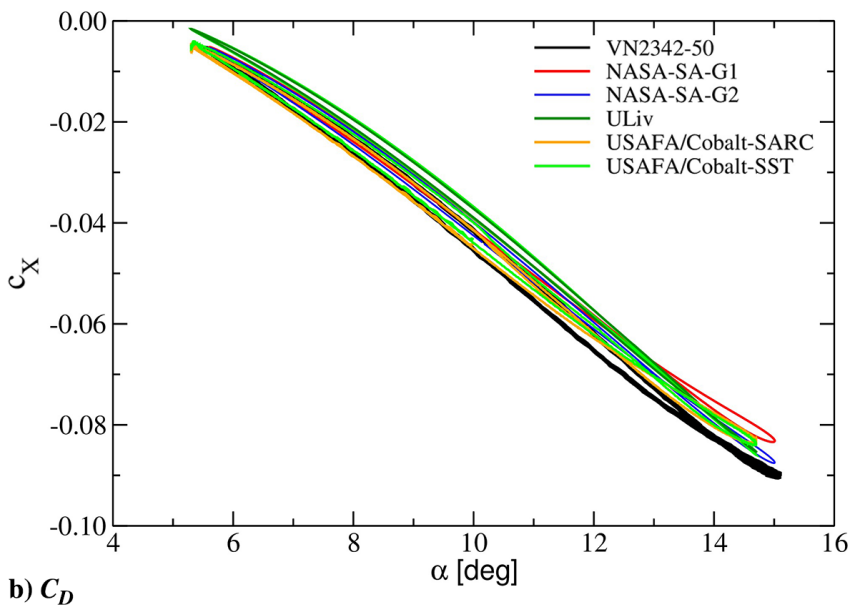

b) $C_{D}$

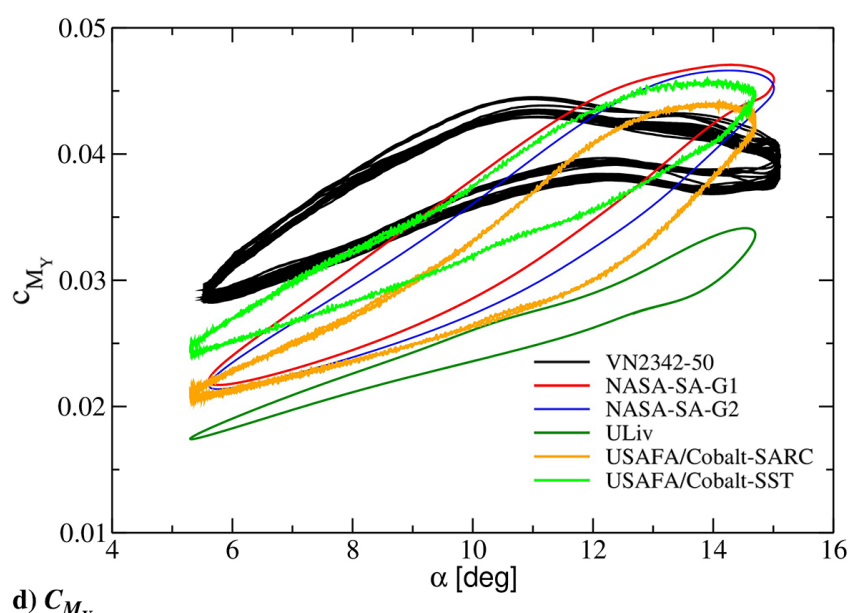

Fig. 15 Comparison of dynamic pitch oscillations at an angle of attack of $10 \pm 5 \mathrm{deg}, f=1 \mathrm{~Hz} ; 2342-2350, M=0.15, R e=1.56 \times 10^{6}$.

attempt is made to evaluate the value provided to the aircraft design community by using such tools.

1) For static cases, there is a clear trend that is consistent with findings from the NATO RTO AVT-161 Task Group, which is that the codes have a tendency to correctly predict the character of the pitch moment curve at angles of attack above $18 \mathrm{deg}$ but do have a problem in correctly predicting the tendency of the lift curve, and vice versa. This is clearly independent of the type of CFD solver and turbulent model that are used. This is a surprising phenomenon and should be investigated further. This may not be a CFD codes/schemes/ turbulence models issue, but it could be due to the full impact of the experimental setup that may not be modeled in the CFD, such as the inclusion of wind-tunnel walls.

2) All codes are able to predict the sudden rise of the pitch moment that occurs at an angle of incidence of around $18 \mathrm{deg}$ with varying degrees of accuracy (in terms of correctly predicting the angle of incidence at which this phenomenon occurs); the full range of predicted angles of attack at which this pitch increase phenomenon occurs is almost $5 \mathrm{deg}$. Most codes are able to predict the sudden decrease of the pitch moment (nonlinear dip) that occurs at angles of incidence around $15 \mathrm{deg}$. It is advised to increase the number of samples provided by the CFD code in this particular region of the pitch moment curve due to the very narrow range of angles of attack at which the sudden change of pitch occurs. The codes generally have problems in predicting the beginning of the nonlinear pitch moment behavior, which starts at an angle of attack around $11 \mathrm{deg}$. This phenomenon is also possibly dependent on the presence of the windtunnel walls in the CFD mesh.

The results of the pitch moment predictions are possibly of an indicative character, i.e., the accuracy of the results is low, however the phenomena is predicted. Such a capability should not be disregarded, but it can be used as a valuable tool for indications of what the experimental resources should be focusing on.
3) The differences between codes increase substantially at angles of incidence above $20 \mathrm{deg}$, as indicated by the large standard deviation of the computational results, which is possibly due to the flow in this region being highly unsteady; any steady analysis will therefore introduce additional uncertainty due to the omission of the temporal characteristics of the steady physical approximation.

4) Predictions of drag are generally very good. For example, at angles of incidence of $10 \mathrm{deg}$, most codes are within a 15-drag-count band from the wind-tunnel data (around $4 \%$ of the value of the drag at this angle of incidence) for geometries both with and without control deflections. Some of the codes predict values of drag accurately, even at very high angles of incidence around $30 \mathrm{deg}$. The only exception is around an angle of attack of $16 \mathrm{deg}$, where the results of the different numerical codes show a larger spread of predicted values.

5) All codes are run in fully turbulent mode. The largest discrepancies take place at higher angles of incidence, where the assumption of fully turbulent flow is most probably valid. It would be beneficial, however, to have the results of a formal study of the boundary-layer transition because transition dots may not yield consistent results at all angles of attack. Furthermore, it is not known to what extent the laminar boundary layer affects the pitch moment predictions at angles below $11 \mathrm{deg}$ because transition is forced at locations after the leading edge of the aircraft.

6) Most codes predict control effectiveness fairly well: in particular, at the lower angles of attack. Therefore, it is highly recommended to use CFD codes to assess control effectiveness, even within the linear portion of the force and moment curves, before any model manufacturing. It is advised to provide a case of symmetric control deflections for evaluation of the numerical code capability to predict the extent of lift and pitch control by different control surfaces.

7) Common cases presented in this paper are tested in the range of angles of attack where the flow is weakly nonlinear. In most cases, the results are obtained using an unsteady Reynolds-averaged 

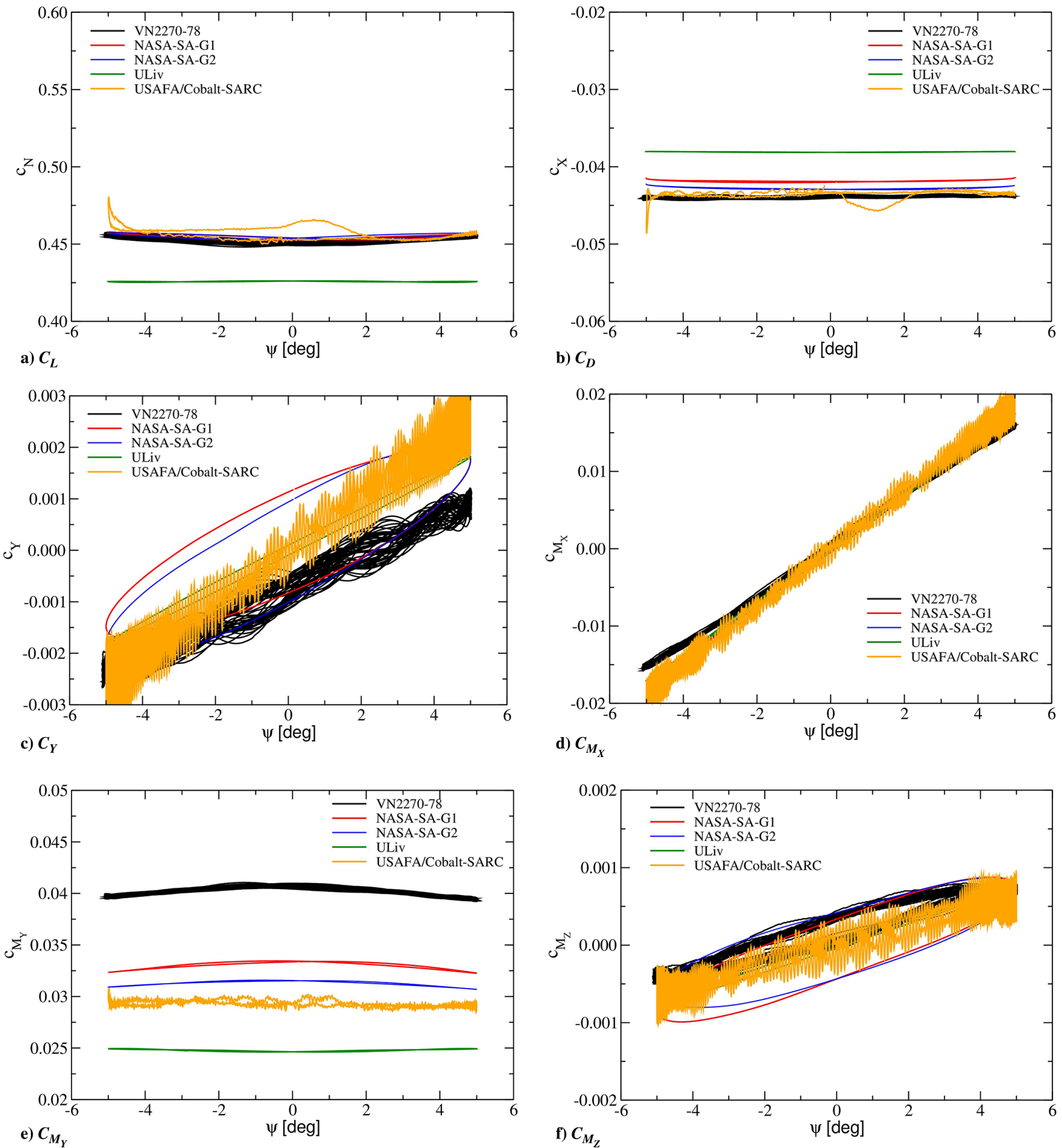

Fig. 16 Comparison of dynamic yaw oscillations at an angle of sideslip of $0 \pm 5 \mathrm{deg}, f=1 \mathrm{~Hz} ; 2270-2278, M=0.15, R e=1.56 \times 10^{6}$.

Navier-Stokes approach, which explains the missing high-frequency content in the CFD results. None of the computations exactly duplicate the nonlinearity for the pitch motion at angles above $11 \mathrm{deg}$; this is consistent with the inability of CFD solvers to predict similar nonlinearity in the static pitch moment data. Although some of these discrepancies have been isolated (such as the pitch moment offset at low angles of attack being due to accurate temporal modeling of the sting flowfield), all other grid/turbulence model issues should be isolated and understood in the future.

Although these assessments do not explain why certain aerodynamic characteristics are not well predicted (such as the nonlinear behavior of the pitching moment), the results do point to certain aspects of the numerical modeling approach that need improvement. Specifically, grid resolution around the leading edge is critical for accurate prediction of the initiation and movement of the vortices on this configuration. Also, turbulence models do not include the ability to accurately predict vortex formation for round leading edges, and this shortcoming should be addressed further. Finally, a number of effects that were due to not modeling the wind tunnel accurately (sting effects and wall effects) were seen; although including these features can significantly increase the cost of performing simulations, their impact can be significant. All of these modeling issues need to be addressed in order to accurately predict the flow over configurations like SACCON, and the results presented here show the need to improve the ability to predict turbulence and accurately model the wind-tunnel environment. 


\section{Acknowledgments}

The authors would like to thank the members of the North Atlantic Treaty Organization's Science and Technology Organisation Task Group Applied Vehicle Technology (AVT)-201 for their hard work and dedication to this project. The breadth of the wind-tunnel tests during AVT-201 has been outstanding, with low- and high-speed subsonic testing taking place at numerous wind tunnels by DLR, German Aerospace Center; BAE Systems; The Defence Science and Technology Laboratory (DSTL); and ONERA-The French Aerospace Lab. The data collected during these tests, both static and dynamic, are the key to the success of AVT-201's goal of determining how well computational fluid dynamics can simulate the stability and control characteristics of the nonlinear aerodynamics of a generic unmanned combat aerial vehicle. In addition, the authors are thankful for the numerous members of the Task Group who have performed challenging numerical simulations of these flows, as well as for everyone involved in creating stability and control databases and models, either from wind-tunnel results or numerical simulations.

\section{References}

[1] Chambers, J. R., and Hall, R. M., "Historical Review of Uncommanded Lateral-Directional Motions at Transonic Conditions," Journal of Aircraft, Vol. 41, No. 3, May-June 2004, pp. 436-447. doi: $10.2514 / 1.4470$

[2] Hall, R. M., Woodson, S. H., and Chambers, J. R., "Accomplishments of the Abrupt-Wing-Stall Program," Journal of Aircraft, Vol. 42, No. 3, May-June 2005, pp. 653-660. doi: $10.2514 / 1.3631$

[3] Hall, R. M., Biedron, R. T., Ball, D. N., Bogue, D. R., Chung, J., Green, B. E., and Chambers, J. R., "Computational Methods for Stability and Control (COMSAC): The Time Has Come," AIAA Atmospheric Flight Mechanics Conference and Exhibit, AIAA Paper 2005-6121, Aug. 2005.

[4] McDaniel, D. R., Cummings, R. M., Bergeron, K., Morton, S. M., and Dean, J. P., "Comparisons of CFD Solutions of Static and Maneuvering Fighter Aircraft with Flight Test Data," 3rd International Symposium on Integrating CFD and Experiments in Aerodynamics, U.S. Air Force Academy, Colorado Springs, CO, June 2007.

[5] Yurkovich, R., and Chen, P. C., "State-of-the-Art of Unsteady Aerodynamics for High Performance Aircraft," 37th AIAA Aerospace Sciences Meeting and Exhibit, AIAA Paper 2001-0428, Jan. 2001.

[6] Meyn, L. A., and James, K. D., "Full Scale Wind Tunnel Studies of F/A-18 Tail Buffet," Journal of Aircraft, Vol. 33, No. 3, May-June 1996, pp. 589-595. doi: $10.2514 / 3.46986$

[7] Jacobson, S. B., Britt, R. T., Freim, D. R., and Kelly, P. D., "Residual Pitch Oscillation Flight Test Analysis on the B-2 Bomber," AIAA Paper 1998-1805, Jan. 1998.

[8] "Assessment of Stability and Control Prediction Methods for NATO Air and Sea Vehicles (AVT-161)," NATO RTO-TR-AVT-161, 2007.

[9] Ball, R. E., The Fundamentals of Aircraft Combat Survivability Analysis and Design, 2nd ed., AIAA Education Series, AIAA, Reston, VA, 2003.

[10] Cummings, R. M., and Schütte, A., "Aerodynamics and Conceptual Design Studies on an Unmanned Combat Aerial Vehicle Configuration," Journal of Aircraft, Nov. 2016. doi:10.2514/1.C033808

[11] Liersch, C. M., and Huber, K. C., "Conceptual Design and Aerodynamic Analyses of a Generic UCAV Configuration," 32nd AIAA Applied Aerodynamics Conference, AIAA Paper 2014-2001, June 2014.

[12] Huber, K. C., Vicroy, D. D., Schuette, A., and Hübner, A., "UCAV Model Design and Static Experimental Investigations to Estimate Control Device Effectiveness and S\&C Capabilities," 32nd AIAA Applied Aerodynamics Conference, AIAA Paper 2014-2002, June 2014.

[13] Vicroy, D. D., Huber, K. C., Loeser, T. D., and Rohlf, D., "Experimental Investigations of a Generic Swept Unmanned Combat Air Vehicle with Controls," Journal of Aircraft (advance for online publication).

[14] Rein, M., Irving, J., Rigby, G., and Birch, T. J., "High Speed Static Experimental Investigations to Estimate Control Device Effectiveness and S\&C Capabilities," 32nd AIAA Applied Aerodynamics Conference, AIAA Paper 2014-2004, June 2014.

[15] Zimper, D., and Rein, M., "Experimental and Numerical Analysis of the Transonic Vortical Flow over a Generic Lambda Wing Configuration," 32nd AIAA Applied Aerodynamics Conference, AIAA Paper 20142005, June 2014.
[16] Schütte, A., Huber, K. C., and Boelens, O. J., "Static and Dynamic Numerical Simulations of a Generic UCAV Configuration with and Without Control Devices," 32nd AIAA Applied Aerodynamics Conference, AIAA Paper 2014-2132, June 2014.

[17] Frink, N. T., "Dynamic Stability and Control CFD Investigations of a Generic 53-deg Swept UCAV Configuration," 32nd AIAA Applied Aerodynamics Conference, AIAA Paper 2014-2133, June 2014

[18] Kennett, D. J., Hoholis, G., and Badcock, K. J., "Numerical Simulation of Control Surface Deflections over a Generic UCAV Configuration at Off-Design Flow Conditions," 32nd AIAA Applied Aerodynamics Conference, AIAA Paper 2014-2134, June 2014.

[19] Lynch, E., Abras, J., Crowell, A. R., and Lee, J., "Static and Dynamic CFD Analysis of a Generic Swept Wing UCAV," 53rd AIAA Aerospace Sciences Meeting, AIAA Paper 2015-1504, Jan. 2015.

[20] Coppin, J., and Birch, T. J., "CFD Predictions of Control Effectiveness for a Generic Highly Swept UCAV Configuration," 32nd AIAA Applied Aerodynamics Conference, AIAA Paper 2014-2135, June 2014.

[21] Young, M. E., Ghoreyshi, M., Jirasek, A., and Cummings, R. M., "Prediction and Validation of Aerodynamic Characteristics for a Generic UCAV Configuration with Trailing-Edge Flaps," 32nd AIAA Applied Aerodynamics Conference, AIAA Paper 2014-2136, June 2014

[22] Lofthouse, A. J., Ghoreyshi, M., Jirasek, A., and Cummings, R. M., "Static and Dynamic Simulations of a Generic UCAV Geometry Using the Kestrel Flow Solver," 32nd AIAA Applied Aerodynamics Conference, AIAA Paper 2014-2264, June 2014.

[23] Ghoreyshi, M., Jirasek, A., Cummings, R. M., Ronch, A. D., and Young, M. E., "Validation of Unsteady Aerodynamic Models of a Generic UCAV Using Overset Grids," 32nd AIAA Applied Aerodynamics Conference, AIAA Paper 2014-2265, June 2014.

[24] Zimper, D., and Hummel, D., "Aerodynamic Behavior and Analysis of the Transonic Flow Around a Generic UCAV Configuration," 32nd AIAA Applied Aerodynamics Conference, AIAA Paper 2014-2266, June 2014

[25] Hitzel, S. M., and Zimper, D., "Model Scale and 'Real' Flight of Generic UCAV and Advanced Combat Aircraft-An Industrial Perspective," 32nd AIAA Applied Aerodynamics Conference, AIAA Paper 20142267, June 2014.

[26] Tomac, M. M., Rizzi, A. W., and Perrot, T., "Advanced Engineering Method for Investigating Stability \& Control Characteristics of UCAV Configuration-SACCON," 32nd AIAA Applied Aerodynamics Conference, AIAA Paper 2014-2392, June 2014.

[27] Le Roy, J.-F., Morgand, S., and Farcy, D., "Static and Dynamic Derivatives on Generic UCAV without and with Leading Edge Control," 32nd AIAA Applied Aerodynamics Conference, AIAA Paper 20142391, June 2014.

[28] Tomac, M. M., and Tormalm, M., "Stability and Control Assessment of a Generic UCAV Design Using the Edge Flow Solver," 32nd AIAA Applied Aerodynamics Conference, AIAA Paper 2015-2392, June 2014.

[29] Irving, J., Vicroy, D. D., and Farcy, D., "Development of an Open-Loop Simulation Model of a Generic UCAV Configuration for Stability \& Control Analyses," 32nd AIAA Applied Aerodynamics Conference, AIAA Paper 2014-2393, June 2014.

[30] Jirasek, A., Cummings, R. M., Schuette, A., and Huber, K. C., "The NATO STO AVT-201 Task Group on Extended assessment of Stability an Control Prediction Methods for NATO Air Vehicles: Summary," 32nd AIAA Applied Aerodynamics Conference, AIAA Paper 20142394, June 2014.

[31] Cummings, R. M., and Schütte, A., "Assessment of Stability and Control Prediction Methods for NATO Air and Sea Vehicles," NATOResearch and Technology Organisation/Applied Vehicle Technology (AVT), AVT-161 Task Group Final Rept. RTO-TR-AVT161 AC/323(AVT-161)TP/440, Brussels, Belgium, Sept. 2012.

[32] Schütte, A., Hummel, D., and Hitzel, S. M., "Flow Physics Analyses of a Generic Unmanned Combat Aerial Vehicle Configuration," Journal of Aircraft, Vol. 49, No. 6, Nov.-Dec. 2012, pp. 1638-1651. doi:10.2514/1.C031386

[33] Brandsma, F. J., Kok, J. C., Dol, H. S., and Elsenaar, A., "Leading Edge Vortex Flow Computations and Comparison with DNW-HST Wind Tunnel Data," National Aerospace Lab. NLR-TP-2001-238, Amsterdam, The Netherlands, June 2001.

[34] Jirásek, A., and Cummings, R. M., "SACCON Static and Dynamic Motion Flow Physics Simulations Using COBALT," AIAA 29th Applied Aerodynamics Conference, AIAA Paper 2011-3965, June 2011. 